\title{
Stability and Hopf Bifurcation Analysis on a Nonlinear Business Cycle Model
}

\author{
Liming Zhao and Zhipei Zhao \\ Department of Management and Economics, Tianjin University, Tianjin 300072, China \\ Correspondence should be addressed to Zhipei Zhao; zhaozp@tju.edu.cn
}

Received 27 February 2016; Revised 5 June 2016; Accepted 11 July 2016

Academic Editor: Sergey A. Suslov

Copyright ( 2016 L. Zhao and Z. Zhao. This is an open access article distributed under the Creative Commons Attribution License, which permits unrestricted use, distribution, and reproduction in any medium, provided the original work is properly cited.

\begin{abstract}
This study begins with the establishment of a three-dimension business cycle model based on the condition of a fixed exchange rate. Using the established model, the reported study proceeds to describe and discuss the existence of the equilibrium and stability of the economic system near the equilibrium point as a function of the speed of market regulation and the degree of capital liquidity and a stable region is defined. In addition, the condition of Hopf bifurcation is discussed and the stability of a periodic solution, which is generated by the Hopf bifurcation and the direction of the Hopf bifurcation, is provided. Finally, a numerical simulation is provided to confirm the theoretical results. This study plays an important role in theoretical understanding of business cycle models and it is crucial for decision makers in formulating macroeconomic policies as detailed in the conclusions of this report.
\end{abstract}

\section{Introduction}

Accompanied with the development of an economy, increasingly, mainstream economic research has maintained a watchful eye on nonlinear dynamics theory, because its influence is spreading over both the microeconomic and macroeconomic fields. Economists are devoted to analyzing every crucial phenomenon of an economic system using economic data mining, such as irregular microeconomic fluctuation, erratic macroeconomic fluctuations, irregular economic growth, structural changes, and overlapping waves of economic development. However, to account for the limitations of these data, quantitative analysis techniques such as data mining and data analysis just scratch the surface of an economic system making it difficult for economists to conduct meaningful discussions or theoretical analysis of an economic system. Therefore, the qualitative theory of the ordinary differential equation plays an important role in analyzing macroeconomic operational mechanisms.

Among the various macroeconomic theories, economic cycles have always been an interesting field that has attracted most economists. The fluctuations of macroeconomic variables can reflect the degree of stability of the whole economic system [1-4]. Due to the historic trends of economic globalization, today there is no complete closed economy. Therefore, it is far more practical today for researchers to conduct studies on open economies. An open economy system includes both domestic and foreign economic activities, which produces the greatest influence on each part of a national economy [5-8].

This study focused on a macroeconomic dynamics model of the Kaldorian economic cycle in an open economic system based on a forecasted capital condition. Recently, many conflicting macroeconomic dynamic models have been generated. The classical model was proposed by Kaldor and the mathematical structure of the business cycle based on the Kaldorian concept has been researched extensively [9-11]. However, it must be noted that most of this research was based on closed economic systems with the exception of a few studies such as those of Lorenz, Asada, and a few others. Lorenz [12] considered the model in an open economic situation and expanded its capabilities. His research played an important role in models of business cycles in open economic situations. Lorenz proposed a multinational model (three nations) where these nations were connected by international trade. Using a numerical simulation, Lorenz found that his model would generate chaos. But this study research considered only fixed exchange rate conditions without capital 
movement and the physical capital stock of each nation was considered to be constant although external investment was allowed. In a sense, the model proposed by Lorenz is a short period model.

Asada [13] took another approach to study the Kaldorian business cycle model in an open economy. In Asada's study, the movement of capital was considered and the study investigated an economic system with both fixed and flexible exchange rate conditions. He also considered the physical capital stock variations resulting from corporate investment, which is a fundamental feature of a Kaldorian business cycle model. To avoid a complicated analysis, Asada restricted his analysis to a small open economic system and the degree of capital mobility was an important parameter used to determine the kinetic properties of the system. Based on the work cited, this current study was intended to investigate the Kaldorian business cycle model with a fixed exchange condition, where the speed of market regulation and the degree of capital movement were considered as system parameters to obtain the salient dynamic properties of the model:

$$
\frac{d Y(t)}{d t}=\alpha[C(t)+I(t)+G+J(t)-Y(t)]
$$

where $\alpha$ represents the speed of market regulation and (1) is the Kaldorian adjustment equation for a commodity market. Here, $C(t)=c[Y(t)-T(t)]+C_{0}$, where $(0<c<1$, $\left.C_{0}>0\right)$ represents the normal Keynes consumption function, and $T(t)=\tau Y(t)-T_{0}$, where $\left(0<\tau<1, T_{0}>0\right)$ represents the revenue function. In $(1), J(t)=J[Y(t), \pi](\partial J / \partial Y<0$, $\partial J / \partial \pi>0)$ represents the balance of current account and it is inversely correlated to the net real national income and positively correlated to the exchange rate $d M(t) / d t=p A(t)$ :

$$
\frac{d K(t)}{d t}=I(t)
$$

Equation (2) is the capital accumulation equation. The rate of change of the actual physical capital stock is equal to net actual private investment in physical capital stock.

In formula (1) and (2), $I(t)=I[Y(t), K(t), r(t)]$, where $\partial I / \partial Y>0, \partial I / \partial K<0, \partial I / \partial r<0 . I(t)$ represents the Kaldorian investment function and its meaning is that net investment expenditure can be expressed by the net real national income, the real physical capital stock, and the nominal interest rate, where $r(t)$ is the nominal interest rate.

The balance function of international payments can be defined as follows:

$$
A(t)=J(t)+Q(t) .
$$

In (3), $Q(t)=\beta\left[r(t)-r_{f}-\left(\pi^{c}(t)-\pi(t)\right) / \pi(t)\right], \beta>0$, represents the capital account equation. This equation illustrates the relationship as follows: (1) the balance of capital account and the degree of capital mobility; (2) the differences between domestic interest rate and others; (3) the proportional relation between the differences of expectant exchange rate and the exchange rate. Here $\beta$ represents the degree of capital movement, $\pi^{e}(t)$ represents the expectant exchange rate, and $r_{f}$ represents the foreign nominal interest rate.
To investigate the Kaldorian model in a fixed exchange rate condition, this study assumes that

$$
\begin{aligned}
\pi(t) & =\bar{\pi}, \\
\pi^{e}(t) & =\bar{\pi}, \\
\frac{d M(t)}{d t} & =p A(t) .
\end{aligned}
$$

Equation (4) represents the institutional arrangement of the system in a condition of the fixed exchange rates. In other words, the exchange rate is a special constant. Equation (5) assumes that the expectant exchange rate will remain constant. Equation (6) shows that the money supply is constantly in flux accompanied by the total international balance of payments. From (1), (2), and (6), a 3-dimensional nonlinear dynamic system can be obtained which is expressed as follows:

$$
\begin{aligned}
& \frac{d Y(t)}{d t}=\alpha[(c-c \tau-1) Y+I(Y, K, r)+J(Y, \bar{\pi})+G \\
& \left.\quad+c T_{0}+C_{0}\right], \\
& \frac{d K(t)}{d t}=I(Y, K, r), \\
& \frac{d M(t)}{d t}=J(Y, \bar{\pi})+\beta\left[r(Y, M)-r_{f}\right] .
\end{aligned}
$$

According to the research of Asada [13], the nominal interest rate function $r(t)$ can be used in the investment functions $I(Y, K, M)$ and $I(Y)$ yielding

$$
\begin{aligned}
r(Y, M) & =\delta \sqrt{Y}-\beta_{2} M, \\
I(Y, K, r) & =I(Y)-d_{1} K-\beta_{1} r, \\
I(Y) & =\gamma \sqrt{Y} .
\end{aligned}
$$

Then system (7) is transformed into the form

$$
\begin{aligned}
& \frac{d Y(t)}{d t}=\alpha\left[(c(1-\varepsilon)-1) Y(t)+\left(\gamma-\beta_{1} \delta\right) \sqrt{Y(t)}\right. \\
& \left.\quad-d_{1} K(t)+\beta_{1} \beta_{2} M(t)+c T_{0}+C_{0}+G\right], \\
& \frac{d K(t)}{d t}=\left(\gamma-\beta_{1} \delta\right) \sqrt{Y(t)}-d_{1} K(t)+\beta_{1} \beta_{2} M(t), \\
& \frac{d M(t)}{d t}=\delta_{1} Y+\beta\left(\delta \sqrt{Y(t)}-\beta_{2} M-r_{0}\right),
\end{aligned}
$$

where $Y$ is actual gross domestic product, $C$ is actual consume expenditure, $K$ is the physical capital stock, $T$ is the actual income tax, $G$ is government expenditures, $M$ is nominal currency supply, $\alpha$ is the market adjustment coefficient, and $\beta$ is the degree of capital movement. 
Furthermore, if we denote $m=c(1-\varepsilon)-1, n=\gamma-\beta_{1} \delta$, $b=\beta_{1} \beta_{2}, a=c T_{0}+C_{0}+G$, then the model can be transferred to

$$
\begin{aligned}
& \frac{d Y(t)}{d t} \\
& \quad=\alpha\left(m Y(t)+n \sqrt{Y(t)}-d_{1} K(t)+b M(t)+a\right), \\
& \frac{d K(t)}{d t}=n \sqrt{Y(t)}-d_{1} K(t)+b M(t), \\
& \frac{d M(t)}{d t}=\delta_{1} Y(t)+\beta\left(\delta \sqrt{Y(t)}-\beta_{2} M(t)-r_{0}\right) .
\end{aligned}
$$

It is well known that $Y(t)$ represents the gross domestic product and it is always positive. Hence, we can assume that $Y(t)>0$, and the function $\sqrt{Y(t)}$ is a smooth function in $(0,+\infty)$. Model $(10)$ has right-hand sides of class $C^{1}(0,+\infty)$.

The paper is organized as follows: in Section 2, the local stability of the equilibrium point of system is described (10), and the market adjustment coefficient $\alpha$ and the degree of capital movement $\beta$ are the system parameters to determine the stable region of the system. In Section 3, the existence of Hopf bifurcation and the stability of the periodic solution generated by Hopf bifurcation based on parameters $\alpha$ and $\beta$ are discussed. Finally, some conclusions are given.

\section{Analysis on Equilibrium State of the System}

2.1. Existence of Equilibrium Point and the Stability of the System. To find the stable economic growth path and obtain the relationship between economic growth, capital accumulation, and nominal currency supply, the equilibrium point of system (10) must be determined as follows:

$$
\begin{array}{r}
\alpha\left(m Y(t)+n \sqrt{Y(t)}-d_{1} K(t)+b M(t)+a\right)=0, \\
n \sqrt{Y(t)}-d_{1} K(t)+b M(t)=0, \\
\delta_{1} Y(t)+\beta\left(\delta \sqrt{Y(t)}-\beta_{2} M(t)-r_{0}\right)=0 .
\end{array}
$$

Therefore, we can obtain the equilibrium point as $E\left(Y^{*}\right.$, $\left.K^{*}, M^{*}\right)$, where

$$
\begin{aligned}
Y^{*} & =-\frac{a}{m}, \\
K^{*} & =\frac{b \delta_{1} Y^{*}+\left(b \delta+n \beta_{2}\right) \beta \sqrt{Y^{*}}-b \beta r_{0}}{\beta \beta_{2} d_{1}}, \\
M^{*} & =\frac{\delta_{1} Y^{*}+\beta\left(\delta \sqrt{Y^{*}}-r_{0}\right)}{\beta \beta_{2}} .
\end{aligned}
$$

If the actual conditions of the system are considered, the physical capital stock $K$, the nominal currency supply $M$, and the gross domestic product $Y$ should be positive values. Hence, the following hypothesis $\left(H_{1}\right)$ is required:
$\left(H_{1}\right)$

$$
\begin{aligned}
c(1-\varepsilon) & <1, \\
\frac{b \delta_{1} Y^{*}+\left(b \delta+n \beta_{2}\right) \beta \sqrt{Y^{*}}-b \beta r_{0}}{\beta \beta_{2} d_{1}} & >0, \\
\frac{\delta_{1} Y^{*}+\beta\left(\delta \sqrt{Y^{*}}-r_{0}\right)}{\beta \beta_{2}} & >0 .
\end{aligned}
$$

When all of parameters meet the hypothesis $\left(H_{1}\right)$, the equilibrium point $E\left(Y^{*}, K^{*}, M^{*}\right)$ of system $(10)$ is positive.

To determine the stability of system near the equilibrium point $E\left(Y^{*}, K^{*}, M^{*}\right)$, according to the theory of nonlinear dynamics, the linear transformation should be considered as follows to move the equilibrium point to the original point:

$$
\begin{aligned}
& x=Y-Y^{*}, \\
& y=K-K^{*}, \\
& z=M-M^{*} .
\end{aligned}
$$

Then, near original point, system (10) can be transferred into the form that

$$
\begin{aligned}
& \frac{d X}{d t}=\alpha\left(n \sqrt{X+Y^{*}}+m X-n \sqrt{Y^{*}}-d_{1} Y+b Z\right), \\
& \frac{d Y}{d t}=n \sqrt{X+Y^{*}}-n \sqrt{Y^{*}}-d_{1} Y+b Z, \\
& \frac{d Z}{d t}=\delta_{1} X+\beta\left(\delta \sqrt{X+Y^{*}}-\delta \sqrt{Y^{*}}-\beta_{2} Z\right) .
\end{aligned}
$$

The Jacobian matrix of system (15) is

$$
\left(\begin{array}{ccc}
\alpha m+\frac{n}{2 \sqrt{Y^{*}}} & -\alpha d_{1} & \alpha b \\
\frac{n}{2 \sqrt{Y^{*}}} & -d_{1} & b \\
\delta_{1}+\frac{\beta \delta}{2 \sqrt{Y^{*}}} & 0 & -\beta \beta_{2}
\end{array}\right) .
$$

And its characteristic polynomial is

$$
f(\lambda)=\lambda^{3}+A \lambda^{2}+B \lambda+C,
$$

where

$$
\begin{aligned}
A= & d_{1}+\beta \beta_{2}-\alpha m-\frac{\alpha n}{2 \sqrt{Y^{*}}}, \\
B= & \beta \beta_{2} d_{1}-\alpha\left(m \beta \beta_{2}-b \delta_{1}-m d_{1}\right) \\
& \quad-\frac{\alpha \beta\left(b \delta+n \beta_{2}\right)}{2 \sqrt{Y^{*}}}, \\
C= & -\alpha m \beta \beta_{2} d_{1} .
\end{aligned}
$$

According to the Routh-Hurwitz criterion, we can obtain the hypothesis: 
$\left(H_{2}\right)$

$$
\begin{aligned}
& A>0, \\
& A B-C>0,
\end{aligned}
$$$$
\text { all roots of the equation } f(\lambda)
$$$$
=0 \text { has strictly negative real part. }
$$

According to the theory of nonlinear dynamics, when the system meets hypotheses $H_{1}, H_{2}$, the equilibrium point $E\left(Y^{*}\right.$, $\left.K^{*}, M^{*}\right)$ is stable.

2.2. Numerical Simulation of Equilibrium Point Stability. To attain the dynamic evolution behaviors and the complexity of system (10), system (10) should be considered with the following parameters based on the former theoretical analysis. According to relevant research [13] we take $c=0.8, \varepsilon=0.425$, $T_{0}=10, C_{0}=70, G=30, r_{0}=6, \delta=10, \beta_{1}=1, \beta_{2}=1$, $d_{1}=0.3, \gamma=25$. And model (10) can be transferred into

$$
\begin{aligned}
\frac{d Y}{d t} & =\alpha(-0.66 Y+15 \sqrt{Y}-0.3 K+M+108), \\
\frac{d K}{d t} & =15 \sqrt{Y}-0.3 K+M, \\
\frac{d M}{d t} & =-0.3 Y+10 \beta \sqrt{Y}-\beta M-6 \beta+50 .
\end{aligned}
$$

System (20) has unique equilibrium point $E\left(Y^{*}, K^{*}, M^{*}\right)$ :

$$
\begin{aligned}
Y^{*} & =163.636, \\
K^{*} & =1046.004+\frac{3.030}{\beta}, \\
M^{*} & =121.920+\frac{0.909}{\beta} .
\end{aligned}
$$

From the matrix (16), the Jacobian matrix of system (20) at origin point is

$$
\left(\begin{array}{ccc}
-0.0736980299 \alpha & -0.3 \alpha & \alpha \\
0.5863019701 & -0.3 & 1 \\
-0.3+0.3908679800 \beta & 0 & -\beta
\end{array}\right) .
$$

And its characteristic polynomial is

$$
\begin{aligned}
f(\lambda)= & \lambda^{3}+(\beta+0.3+0.074 \alpha) \lambda^{2} \\
& +((-0.317 \beta+0.498) \alpha+0.3 \beta) \lambda \\
& +0.198 \alpha \beta .
\end{aligned}
$$

According to (17),

$$
\begin{aligned}
& A=\beta+0.3+0.074 \alpha, \\
& B=(-0.317 \beta+0.498) \alpha+0.3 \beta, \\
& C=0.198 \alpha \beta .
\end{aligned}
$$

Thus, according to the Routh-Hurwitz criterion and hypothesis (19), we know that the equilibrium point $E\left(Y^{*}, K^{*}, M^{*}\right)$ of the system needs to meet $A B>C$; that is,

$$
\begin{aligned}
\varphi_{s}= & -0.317 \alpha \beta^{2}+0.227 \alpha \beta+0.3 \beta^{2}+0.149 \alpha+0.09 \beta \\
& -0.023 \alpha^{2} \beta+0.037 \alpha^{2}>0 .
\end{aligned}
$$

From the plane $\alpha-\beta$, we can obtain the stable boundary curve $\varphi_{s}=0$ and the stable region $\varphi_{s}>0$, whose parameters, $\alpha \in[1,3]$ and $\beta \in[1.5,10]$, are shown in Figure 1 . At the same time, the stable critical surface in three-dimensional space $\varphi_{s^{-}}$ $\alpha-\beta$ can be obtained. The area under the stable critical surface represents the stable region, and inversely, the area above it represents the unstable region as shown in Figure 2.

The solid line in Figure 1 represents the critical curve $\varphi_{s}=$ 0 through which the status changes from stable to unstable at the equilibrium point $E\left(Y^{*}, K^{*}, M^{*}\right)$ and vice versa. When parameters of system (20), as shown from the gray portion $\left(\varphi_{s}>0\right)$ in Figure 1, passes through the boundary curve into the white part, system (20) becomes unstable. That is to say, no matter the initial value, so long as the parameters pair $(\alpha, \beta)$ is in the gray area of the curve, the time histories of the gross domestic product $Y$, the physical capital stock $K$, and the nominal currency supply $M$ will converge to the equilibrium point after initial oscillation, and the phase diagram of economic system will gradually converge to the equilibrium point.

However, when the parameter pair $(\alpha, \beta)$ assumes a position at the point in white part of the curve, the history of the gross domestic product $Y$, the physical capital stock $K$, and the nominal currency supply $M$ cannot converge to equilibrium. They may converge to a close orbit (limit cycle) or emerge in a diverging status, to the point where they emerge to an irregular thermal agitation status (chaos status), and the phase diagram never converges to the equilibrium point. Two points were extracted from these two areas and placed into system (20) to observe the changes in the history and phase diagram shown in Figures 3 and 4.

\section{Existence and Stability of Hopf Bifurcation}

As is previously mentioned, as the parameters $\alpha, \beta$ change, the system passes through a critical curve into an unstable status. The numerical simulation in last section shows that the unstable status presents different phenomena such as the status of divergence, converging to a closed orbit, or passing to a chaos. Furthermore, especially with economic systems, if the system is stable, the solution should converge to the equilibrium point shown in Figure 3. However, when the system becomes unstable near the equilibrium $E\left(Y^{*}, K^{*}, M^{*}\right)$, the changes in the system are an important area of interest. When the system converges to a closed limit cycle, each variable of the system assumes periodic variations. In the history of the system variables, the amplitude will exhibit periodic oscillations. This phenomenon can be explained by how the business cycle is generated, which includes the economic preparation period, growth period, recession period, and adjustment period. Hopf bifurcation can help to explain these periodic variations in an economic system. In this section the 


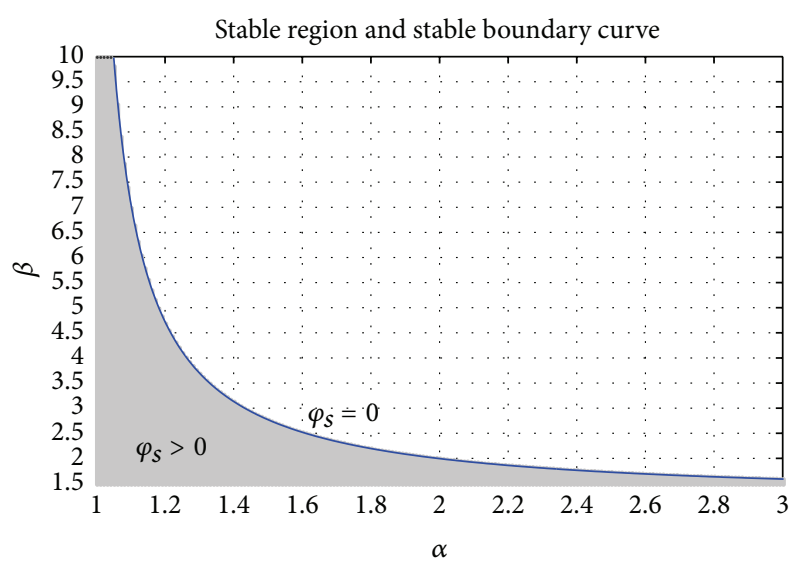

FIGURE 1: Stable region $\varphi_{s}=0$ and stable boundary curve $\varphi_{s}=0$.

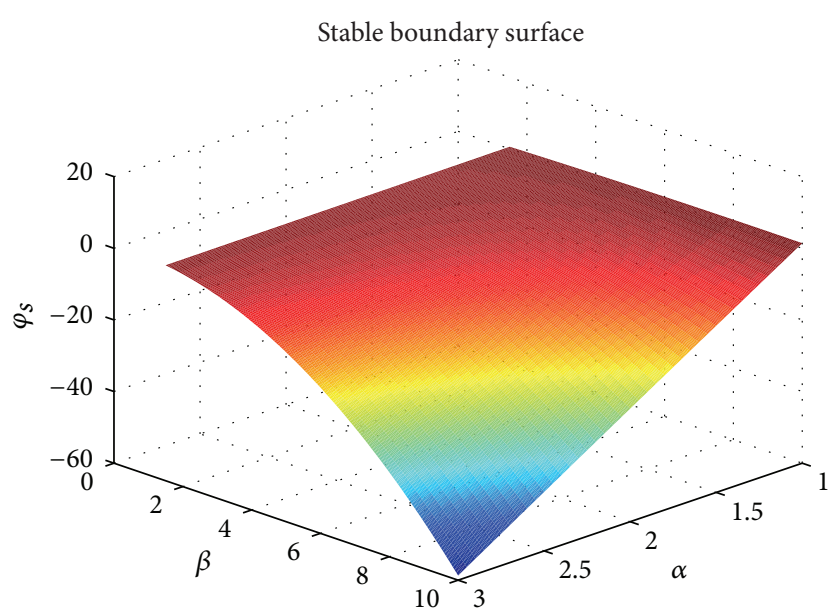

FIGURE 2: Stable critical surface $\varphi_{s}$.

existence and stability of the periodic solution generated by the Hopf bifurcation of the system with the parameters $\alpha$ and $\beta$ will be investigated.

3.1. Existence of Hopf Bifurcation. According to the theory of nonlinear dynamics, if the characteristic equation of system (15) has a pair of conjugate purely imaginary characteristic roots, a Hopf bifurcation of system (15) occurs. The bifurcation equation is

$$
\lambda^{3}+A \lambda^{2}+B \lambda+C=0 .
$$

Assuming that $\lambda_{1,2}= \pm i \omega_{0}$ is a pair of conjugate purely imaginary characteristic roots and substituting $\lambda=i \omega$ into the characteristic polynomial as well as letting $f(\omega)=0$, we obtain

$$
\begin{aligned}
B \omega-\omega^{3} & =0, \\
-A \omega^{2}+C & =0 ;
\end{aligned}
$$

then $\omega=\sqrt{B}$ and the bifurcation equation is $C-A B=0$; that is,

$$
\begin{aligned}
& \left(d_{1}+\beta \beta_{2}-\alpha m-\frac{\alpha n}{2 \sqrt{Y^{*}}}\right)\left(\beta \beta_{2} d_{1}\right. \\
& \left.-\alpha\left(m \beta \beta_{2}-b \delta_{1}-m d_{1}\right)-\frac{\alpha \beta\left(b \delta+n \beta_{2}\right)}{2 \sqrt{Y^{*}}}\right) \\
& +\alpha m \beta \beta_{2} d_{1}=0 .
\end{aligned}
$$

Through the differential operation to (26),

$$
3 \lambda^{2} \frac{d \lambda}{d \alpha}+2 \lambda A \frac{d \lambda}{d \alpha}+\lambda^{2} \frac{d A}{d \alpha}+B \frac{d \lambda}{d \alpha}+\lambda \frac{d B}{d \alpha}+\frac{d C}{d \alpha}=0,
$$

denote $e_{1}=d A / d \alpha, e_{2}=d B / d \alpha$, and $e_{3}=d C / d \alpha$, and we obtain

$$
\frac{d \lambda}{d \alpha}=-\frac{\lambda^{2} e_{1}+\lambda e_{2}+e_{3}}{3 \lambda^{2}+2 A \lambda+B},
$$

where $e_{1}=-\left(m+n / 2 \sqrt{Y^{*}}\right), e_{2}=b \delta_{1}+m d_{1}-m \beta \beta_{2}-\beta(b \delta+$ $\left.n \beta_{2}\right) / 2 \sqrt{Y^{*}}$, and $e_{3}=-m \beta \beta_{2} d_{1}$.

Substitute $\lambda=i \omega_{0}$ into the equation, and calculate the real part of $d \lambda / d \alpha$ :

$$
\operatorname{Re}\left(\frac{d \lambda}{d \alpha}\right)=\frac{A e_{2}+B e_{1}-e_{3}}{2\left(A^{2}+B\right)} .
$$

From this analysis, Theorem 1 is obtained with parameter $\alpha$.

Theorem 1. If system (15) simultaneously meets the three conditions shown as

(1) $\left.\operatorname{Re}[d \lambda / d \alpha]\right|_{\alpha=\alpha_{0}} \neq 0$,

(2) $A^{2}+B>0$,

(3) $A e_{2}+B e_{1}-e_{3} \neq 0$,

system (15) then generates a Hopf bifurcation at $\alpha=\alpha_{0}$, and $a$ limit cycle (periodic solution) will appear near the equilibrium point $E^{*}\left(Y^{*}, K^{*}, M^{*}\right)$.

In a similar way, with a fixed $\alpha$, the situation of the system with the parameter $\beta$ is

$$
\frac{d \lambda}{d \beta}=-\frac{\lambda^{2} f_{1}+\lambda f_{2}+f_{3}}{3 \lambda^{2}+2 A \lambda+B},
$$

where $f_{1}=d A / d \beta, f_{2}=d B / d \beta$, and $f_{3}=d C / d \beta$ :

$$
\begin{aligned}
& f_{1}=\beta_{2}, \\
& f_{2}=\beta_{2} d_{1}-\alpha m \beta_{2}-\frac{\alpha\left(b \delta+n \beta_{2}\right)}{2 \sqrt{Y^{*}}}, \\
& f_{3}=-m \beta_{2} d_{1} .
\end{aligned}
$$

Substitute $\lambda=i \omega_{0}$ into the equation, and calculate the real part of $d \lambda / d \beta$ :

$$
\operatorname{Re}\left(\frac{d \lambda}{d \beta}\right)=\frac{A f_{2}+B f_{1}-f_{3}}{2\left(A^{2}+B\right)} .
$$

Then we have Theorem 2 shown as follows. 

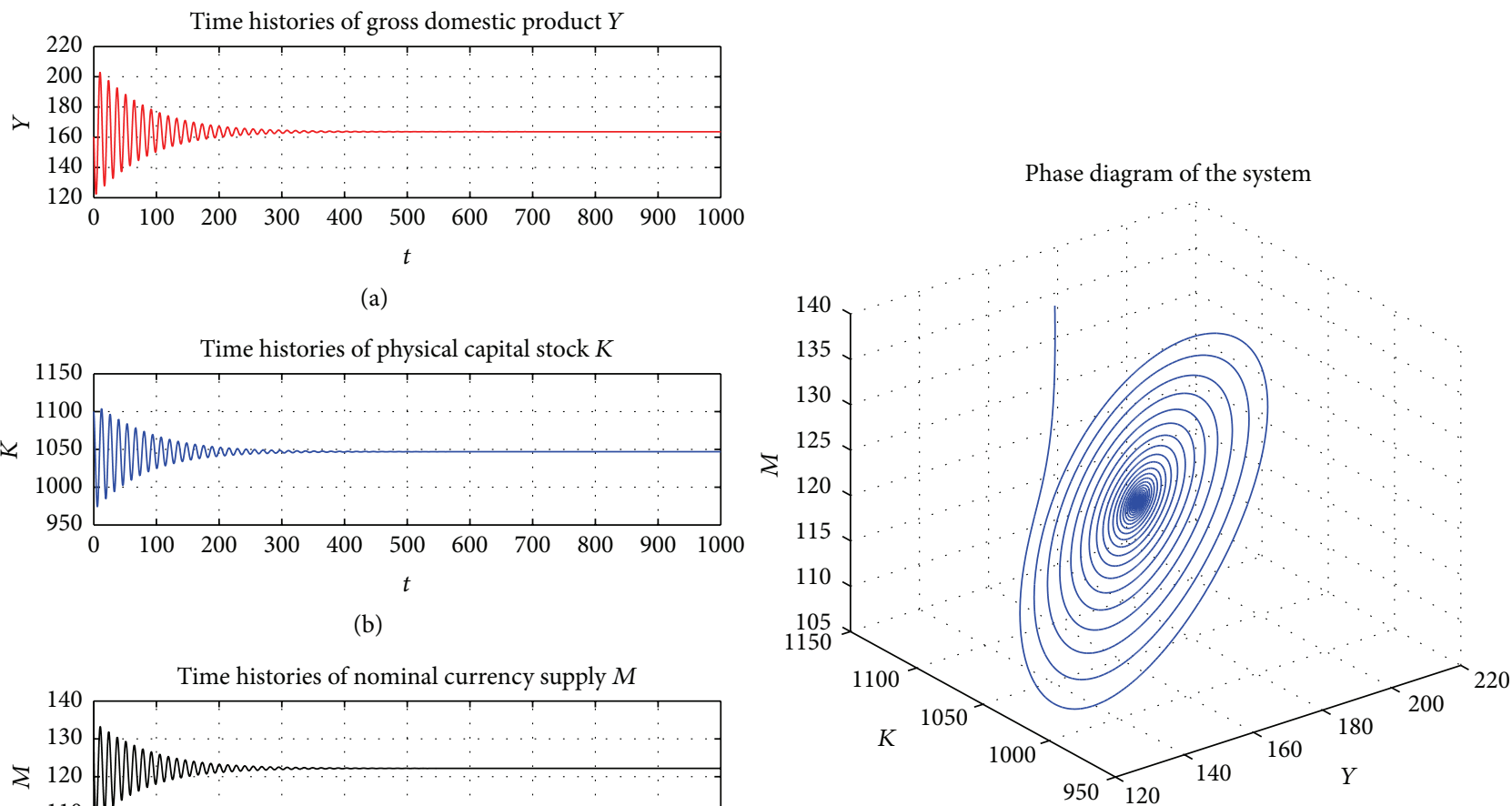

(a)

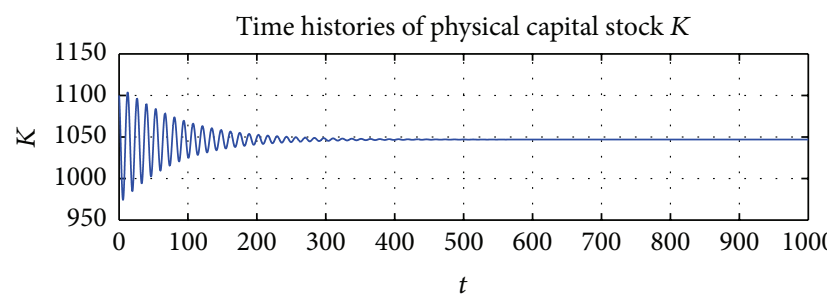

(b)

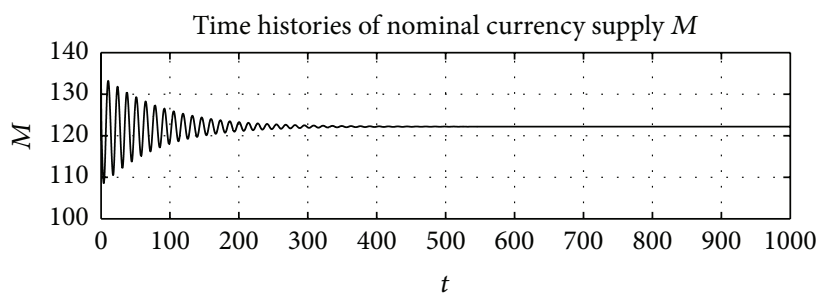

(d)

(c)

FIGURE 3: History and phase diagram of system with $\alpha=1.2, \beta=3.5$.

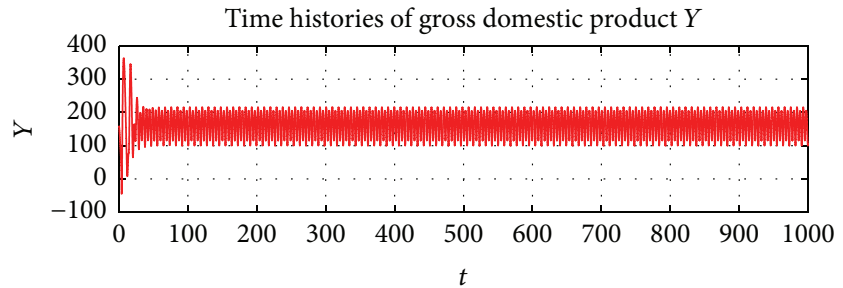

(a)

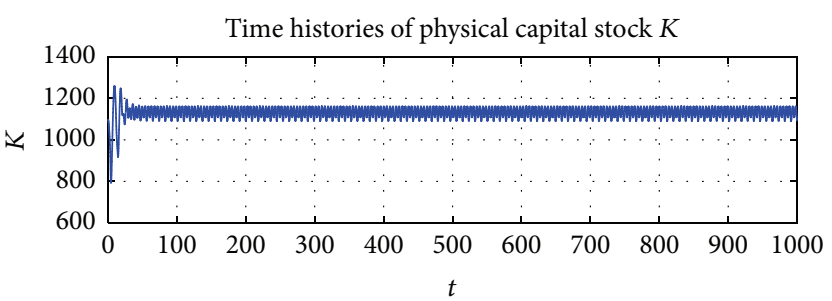

(b)
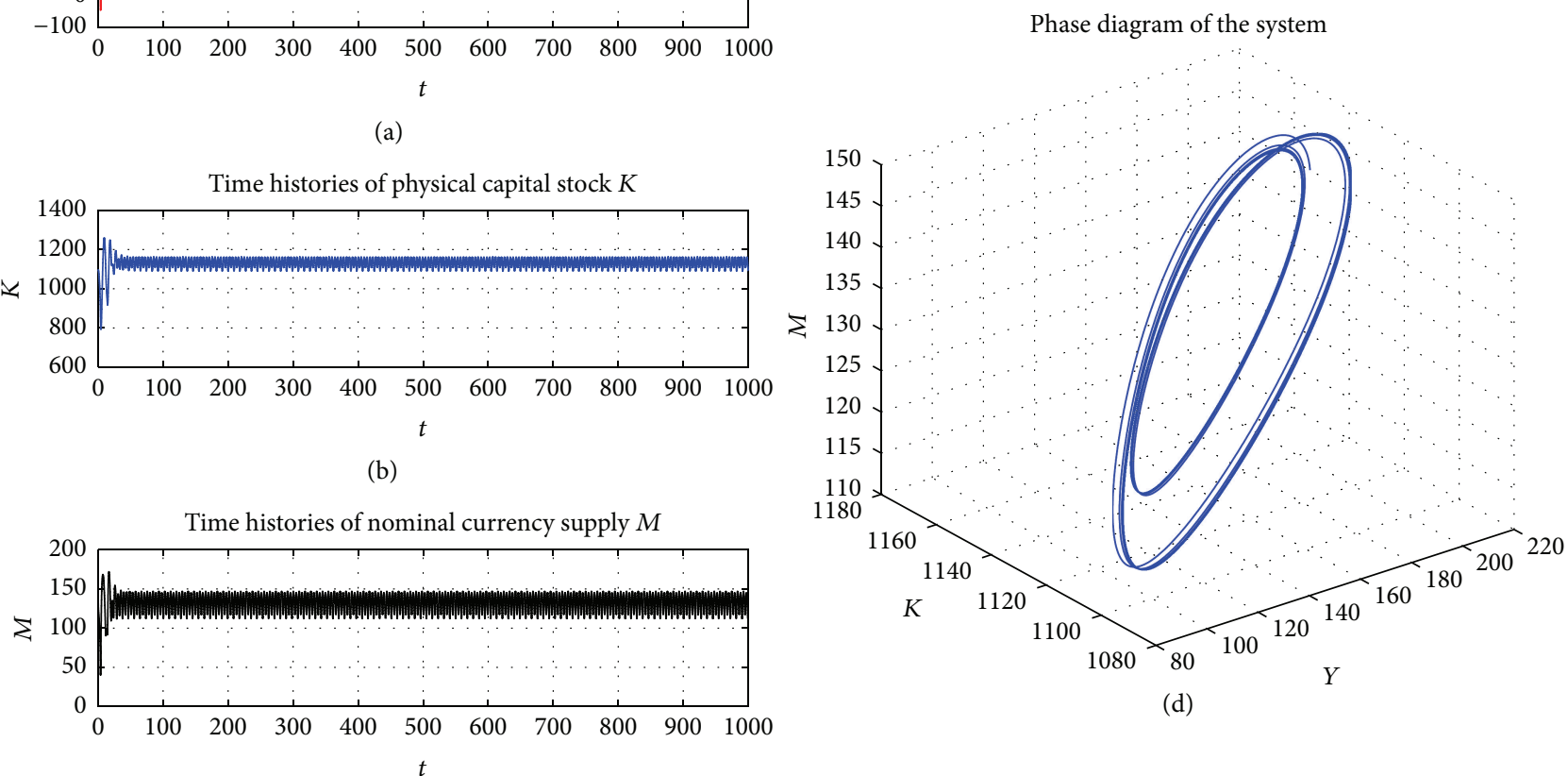

(d)

(c)

FIGURE 4: History and phase diagram of system with $\alpha=2.5, \beta=3.5$. 
Theorem 2. If system (15) simultaneously meets the three conditions shown as

(1) $\left.\operatorname{Re}[d \lambda / d \beta]\right|_{\beta=\beta_{0}} \neq 0$,

(2) $A^{2}+B>0$,

(3) $A f_{2}+B f_{1}-f_{3} \neq 0$,

system (15) then generates a Hopf bifurcation at $\beta=\beta_{0}$, and $a$ limit cycle (periodic solution) will appear near the equilibrium point $E^{*}\left(Y^{*}, K^{*}, M^{*}\right)$.

3.2. Direction and Stability of Hopf Bifurcation. A continuous time nonlinear dynamic system is shown as follows:

$$
\dot{x}=A x+N(x), \quad A=\left(a_{i j}\right)_{n \times n}, x \in \mathbb{R}^{n},
$$

where the smooth function $N(x)=O\left(\|x\|^{2}\right)$ is the nonlinear part of the dynamic system. $N(x)$ can be written into the Taylor series form at origin point, where

$$
N(x)=\frac{1}{2} B(x, x)+\frac{1}{6}(x, x, x)+o\left(\|x\|^{4}\right)
$$

and the $B(x, y)$ and $C(x, y, z)$ are multilinear functions and can be denoted as

$$
\begin{gathered}
B_{i}(x, y)=\left.\sum_{j, k=1}^{2} \frac{\partial^{2} F_{i}(\xi, 0)}{\partial \xi_{j} \partial \xi_{k}}\right|_{\xi=0} x_{j} y_{k} ; \quad i=1,2, \\
C_{i}(x, y, u)=\left.\sum_{j, k=1}^{3} \frac{\partial^{3} F_{i}(\xi, 0)}{\partial \xi_{j} \partial \xi_{k} \partial \xi_{l}}\right|_{\xi=0} x_{j} y_{k} u_{l} ; \quad i=1,2,3 .
\end{gathered}
$$

If matrix $A$ has a unique pair of conjugate purely imaginary characteristic roots $\lambda_{1,2}= \pm i \omega_{0}\left(\omega_{0}>0\right)$, and we denote $q$ as the characteristic vector corresponding to the characteristic value $i \omega_{0}$ of matrix $A$, then

$$
A q=i \omega_{0} q \text {. }
$$

Furthermore, denote $p$ as the characteristic vector corresponding to characteristic value $-i \omega_{0}$ of matrix $A^{T}$, and we have

$$
A^{T} p=-i \omega_{0} p
$$

and $p, q$ meet $\langle q, p\rangle=\bar{q}_{1} p_{2}+\bar{q}_{2} p_{2}+\cdots+\bar{q}_{n} p_{n}=1$, so we have denotations as follows:

$$
\begin{aligned}
g_{20}= & \langle p, B(q, q)\rangle \\
g_{11}= & \langle p, B(q, \bar{q})\rangle \\
g_{21}= & \langle p, C(q, q, \bar{q})\rangle-2\left\langle p, B\left(q, A^{-1} B(q, \bar{q})\right)\right\rangle \\
& +\left\langle p, B\left(\bar{q},\left(2 i \omega_{0} I_{n}-A\right)^{-1} B(q, q)\right)\right\rangle \\
& +\frac{1}{i \omega_{0}}\langle p, B(q, q)\rangle\langle p, B(q, \bar{q})\rangle \\
& -\frac{1}{i \omega_{0}}|\langle p, B(q, \bar{q})\rangle|^{2} \\
& -\frac{1}{3 i \omega_{0}}|\langle p, B(\bar{q}, \bar{q})\rangle|^{2} .
\end{aligned}
$$

Thus, we obtain the unique expression of the first Lyapunov coefficient:

$$
\begin{aligned}
& l_{1}(0)=\frac{1}{2 \omega_{0}} \operatorname{Re}\left(i g_{20} g_{11}+\omega_{0} g_{21}\right)=\frac{1}{2 \omega_{0}} \\
& \cdot \operatorname{Re}\left[\langle p, C(q, q, \bar{q})\rangle-2\left\langle p, B\left(q, A^{-1} B(q, \bar{q})\right)\right\rangle\right. \\
& \left.+\left\langle p, B\left(\bar{q},\left(2 i \omega_{0} Y_{n}-A\right)^{-1} B(q, q)\right)\right\rangle\right] .
\end{aligned}
$$

Accordingly, the Jacobian matrix $A$ at origin point of system (15) is

$$
\left(\begin{array}{ccc}
\alpha m+\alpha n R & -\alpha d_{1} & \alpha b \\
n R & -d_{1} & b \\
\delta_{1}+n \beta \delta & 0 & -\beta \beta_{2}
\end{array}\right),
$$

where $R=1 / 2 \sqrt{Y^{*}}$.

Assuming that matrixes $A$ and $A^{T}$ both have a pair of conjugate purely imaginary characteristic roots $\lambda_{1,2}= \pm i \omega_{0}$, then according to the theory of the symmetric polynomial in complex numbers fields

$$
\begin{aligned}
& \operatorname{trace}(A)=i \omega_{0}-i \omega_{0}+\frac{|A|}{\omega_{0}^{2}} \\
&=\alpha(2 m+n R)-d_{1}-\beta \beta_{2} \\
& \qquad|A|=\alpha \beta m \beta_{2} d_{1} ;
\end{aligned}
$$

then

$$
\omega=\sqrt{\frac{\alpha \beta m \beta_{2} d_{1}}{\alpha(2 m+n R)-d_{1}-\beta \beta_{2}}} .
$$

Through the equations shown as follows,

$$
\begin{aligned}
A q & =i \omega_{0} q, \\
A \bar{q} & =-i \omega_{0} \bar{q}, \\
A^{T} p & =-i \omega_{0} p, \\
A^{T} \bar{p} & =-i \omega_{0} \bar{p},
\end{aligned}
$$

we get the characteristic vector $q$ corresponding to the characteristic value $i \omega_{0}$ of matrix $A$ and the characteristic vector $p$ corresponding to the characteristic value $-i \omega_{0}$ of matrix $A^{T}$. When $q=\left(q_{1}, q_{2}, q_{3}\right)^{T} \neq 0$, the system has a complex solution:

$$
\begin{aligned}
& {\left[\begin{array}{ccc}
-I \omega_{0}+\alpha(R n+m) & -\alpha d_{1} & \alpha n_{1} \\
n R & -I \omega_{0}-d_{1} & n_{1} \\
R m_{1}+\delta_{1} & 0 & -I \omega_{0}-n_{2}
\end{array}\right]\left(\begin{array}{l}
q_{1} \\
q_{2} \\
q_{3}
\end{array}\right)} \\
& \quad=0,
\end{aligned}
$$

and the characteristic polynomial is

$$
\begin{aligned}
& f(\omega)=i \omega^{3}+\left(-R \alpha n-\alpha m+\beta \beta_{2}+d_{1}\right) \omega^{2} \\
& \quad+\left(i R \alpha \beta n \beta_{2}+i \alpha b \beta \delta n+i \alpha \beta m \beta_{2}+i \alpha b \delta_{1}+i \alpha m d_{1}\right. \\
& \left.\quad-i \beta_{2} \beta d_{1}\right) \omega+\alpha \beta m \beta_{2} d_{1}
\end{aligned}
$$


and the characteristic vector $q$ corresponding to the characteristic value $i \omega_{0}$ of matrix $A$ is

$$
\begin{gathered}
q=\left(\frac{-\omega^{2}+i \beta \omega \beta_{2}+i \omega d_{1}+\beta \beta_{2} d_{1}}{i \omega n R+\beta \beta_{2} n R+b \beta \delta n+b \delta_{1}}, 1,\right. \\
\left.\frac{i \beta \delta n \omega+\beta \delta n d_{1}+i \omega \delta_{1}+d_{1} \delta_{1}}{i \omega n R+\beta \beta_{2} n R+b \beta \delta n+b \delta_{1}}\right)^{T} .
\end{gathered}
$$

In a similar way, we can obtain the characteristic $p$ corresponding to the characteristic value $-i \omega$ of matrix $A^{T}$ which is

$$
\begin{aligned}
p & =\frac{1}{\bar{D}}\left(\frac{-i\left(\omega^{2}+i \beta \omega \beta_{2}+i \omega d_{1}-\beta \beta_{2} d_{1}\right)}{\alpha b \omega},\right. \\
& \left.-\frac{d_{1}\left(i \beta_{2} \beta+\omega\right)}{b \omega}, 1\right)^{T} .
\end{aligned}
$$

Because $\langle p, q\rangle=1$, we have

$$
\begin{aligned}
& \bar{D}=\frac{1}{D_{1}}\left(2 i \alpha b^{2} \beta^{2} \delta n \beta_{2} d_{1} \delta_{1}-i R \beta^{3} n \beta_{2}{ }^{3} d_{1}{ }^{2}\right. \\
& -i b \beta^{2} \beta_{2}{ }^{2} d_{1}{ }^{2} \delta_{1}-i b \beta \delta n \omega^{4}-i R \beta n \omega^{2} \beta_{2} d_{1}{ }^{2} \\
& +2 i R \alpha b \beta^{3} \delta n^{2} \beta_{2}{ }^{2} d_{1}+2 i R \alpha b \beta^{2} n \beta_{2}{ }^{2} d_{1} \delta_{1} \\
& +i b \beta \delta n \omega^{2} d_{1}{ }^{2}+i b \beta^{3} \delta n \omega^{2} \beta_{2}{ }^{2}+i \alpha b^{2} \beta \beta_{2} d_{1} \delta_{1}{ }^{2} \\
& +i R^{2} \alpha \beta^{3} n^{2} \beta_{2}^{3} d_{1}-i R \alpha b n \omega^{2} d_{1} \delta_{1} \\
& -i R \alpha b \beta \delta n^{2} \omega^{2} d_{1}+i \alpha b^{2} \beta^{2} \delta^{2} n^{2} \omega^{2}+R \alpha b \beta \delta n^{2} \omega^{3} \\
& +2 b \beta^{3} \delta n \omega \beta_{2}{ }^{2} d_{1}+2 b \beta^{2} \delta n \omega \beta_{2} d_{1}^{2} \\
& -R \alpha b \beta^{2} \delta n^{2} \omega \beta_{2} d_{1}-R \alpha b \beta n \omega \beta_{2} d_{1} \delta_{1} \\
& +i R \alpha b \beta^{2} \delta n^{2} \omega^{2} \beta_{2}+i R \alpha b \beta n \omega^{2} \beta_{2} \delta_{1} \\
& +2 i R \beta^{2} n \omega^{2} \beta_{2}^{2} d_{1}+4 i b \beta \omega^{2} \beta_{2} d_{1} \delta_{1} \\
& +2 i \alpha b^{2} \beta \delta n \omega^{2} \delta_{1}+4 i b \beta^{2} \delta n \omega^{2} \beta_{2} d_{1} \\
& -R^{2} \alpha \beta^{2} n^{2} \omega \beta_{2}^{2} d_{1}+2 i R n \omega^{4} d_{1}-2 b \omega^{3} d_{1} \delta_{1} \\
& +2 R \beta^{3} n \omega \beta_{2}{ }^{3} d_{1}+i R \beta^{3} n \omega^{2} \beta_{2}{ }^{3}+R n \omega^{3} d_{1}{ }^{2} \\
& +i R \beta n \omega^{4} \beta_{2}+2 b \beta \omega \beta_{2} d_{1}{ }^{2} \delta_{1}-R \beta^{2} n \omega^{3} \beta_{2}{ }^{2} \\
& +i R^{2} \alpha \beta n^{2} \omega^{2} \beta_{2} d_{1}+i b \beta^{2} \omega^{2} \beta_{2}^{2} \delta_{1}+R \alpha b n \omega^{3} \delta_{1} \\
& +2 R \beta n \omega^{3} \beta_{2} d_{1}+2 b \beta^{2} \omega \beta_{2}{ }^{2} d_{1} \delta_{1}-2 b \beta \delta n \omega^{3} d_{1} \\
& +R \beta^{2} n \omega \beta_{2}{ }^{2} d_{1}{ }^{2}-2 b \beta^{2} \delta n \omega^{3} \beta_{2}-R n \omega^{5} \\
& -2 b \beta \omega^{3} \beta_{2} \delta_{1}-R^{2} \alpha n^{2} \omega^{3} d_{1}+i \alpha b^{2} \omega^{2} \delta_{1}{ }^{2} \\
& +i b \omega^{2} d_{1}^{2} \delta_{1}+i \alpha b^{2} \omega^{2} \delta_{1}{ }^{2}-i b \beta^{3} \delta n \beta_{2}{ }^{2} \\
& \left.-i b \omega^{4} \delta_{1} d_{1}^{2}+i \alpha b^{2} \beta^{3} \delta^{2} n^{2} \beta_{2} d_{1}\right) \text {, }
\end{aligned}
$$

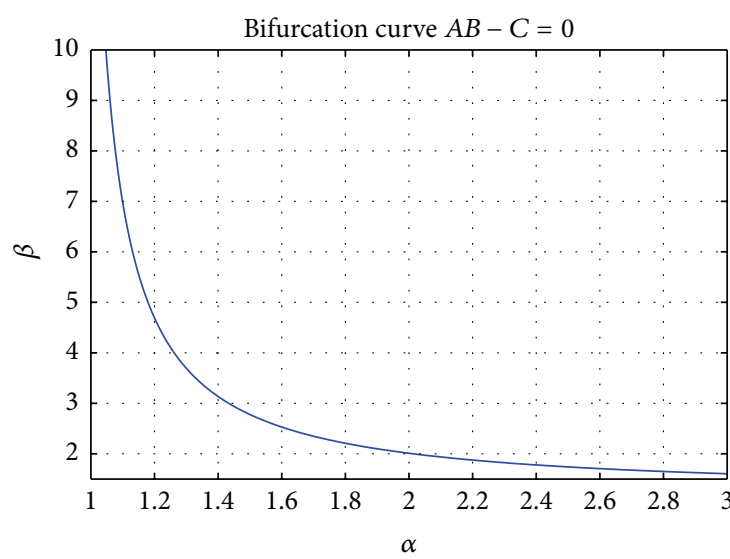

Figure 5: Bifurcation curve diagram.

where $D_{1}=b\left(\left(\beta \delta n+\delta_{1}\right)^{2} b^{2}+2 R n \beta \beta_{2}\left(\beta \delta n+\delta_{1}\right) b+\right.$ $\left.R^{2} n^{2}\left(\beta^{2} \beta_{2}^{2}+\omega^{2}\right)\right) \omega \alpha$.

According to the definition of the linear function we can obtain the bilinear function $B(\xi, \eta)$ and the trilinear function $C(\xi, \eta, \zeta)$ shown as

$$
\begin{gathered}
B(\xi, \eta)=\left(-\frac{\alpha n}{4 Y^{*} \sqrt{Y^{*}}} \xi_{1} \eta_{1}, 0,0\right)^{T}, \\
C(\xi, \eta, \zeta)=\left(\frac{3 \alpha n}{8\left(Y^{*}\right)^{2} \sqrt{Y^{*}}} \xi_{1} \eta_{1} \zeta_{1}, 0,0\right)^{T} .
\end{gathered}
$$

Then we acquire the real part of the first Lyapunov coefficient of system (15), and the expression of $L_{1}(0)$ is

$$
\begin{aligned}
& L_{1}(0)=\frac{1}{2 \omega}\left(\frac{3 \bar{p}_{1} \alpha n q_{1}{ }^{2} \bar{q}_{1}}{\left(Y^{*}\right)^{2} \sqrt{Y^{*}}}-\frac{\bar{p}_{1} n q_{1} \bar{q}_{1}}{2 m Y^{*} \sqrt{Y^{*}}}\right. \\
& -\frac{\bar{p}_{2}\left(R n \beta \beta_{2}+b \beta \delta n+b \delta_{1}\right) n q_{1} \bar{q}_{1}}{2 m \beta \beta_{2} Y^{*} \sqrt{Y^{*}}} \\
& -\frac{\bar{p}_{3}\left(\beta \delta n+\delta_{1}\right) n q_{1} \bar{q}_{1}}{2 m \beta \beta_{2} Y^{*} \sqrt{Y^{*}}} \\
& \left.-\frac{16}{S Y^{* 3}} \alpha^{2} n^{2} \bar{p}_{1} \bar{q}_{1}\left(2 i \omega+d_{1}\right)+\left(2 i \omega+\beta \beta_{2}\right) q_{1}^{2}\right),
\end{aligned}
$$

where

$$
\begin{aligned}
S= & 2 i R \alpha \beta n \omega \beta_{2}+2 i \alpha b \beta \delta n \omega+2 i \alpha \beta m \omega \beta_{2}+2 i \alpha b \omega \delta_{1} \\
& +2 i \alpha m \omega d_{1}-2 i \beta \omega \beta_{2} d_{1}-4 R \alpha n \omega^{2}+\alpha \beta m \beta_{2} d_{1} \\
& +8 i \omega^{3}-4 \alpha m \omega^{2}+4 \beta \omega^{2} \beta_{2}+4 \omega^{2} d_{1} .
\end{aligned}
$$

3.3. Numerical Simulation of Hopf Bifurcation and Its Properties. Continuing the investigation of system (20) and the relevant parameters are the same as in Section 2.2. We will first discuss the existence of Hopf bifurcation. We know the 

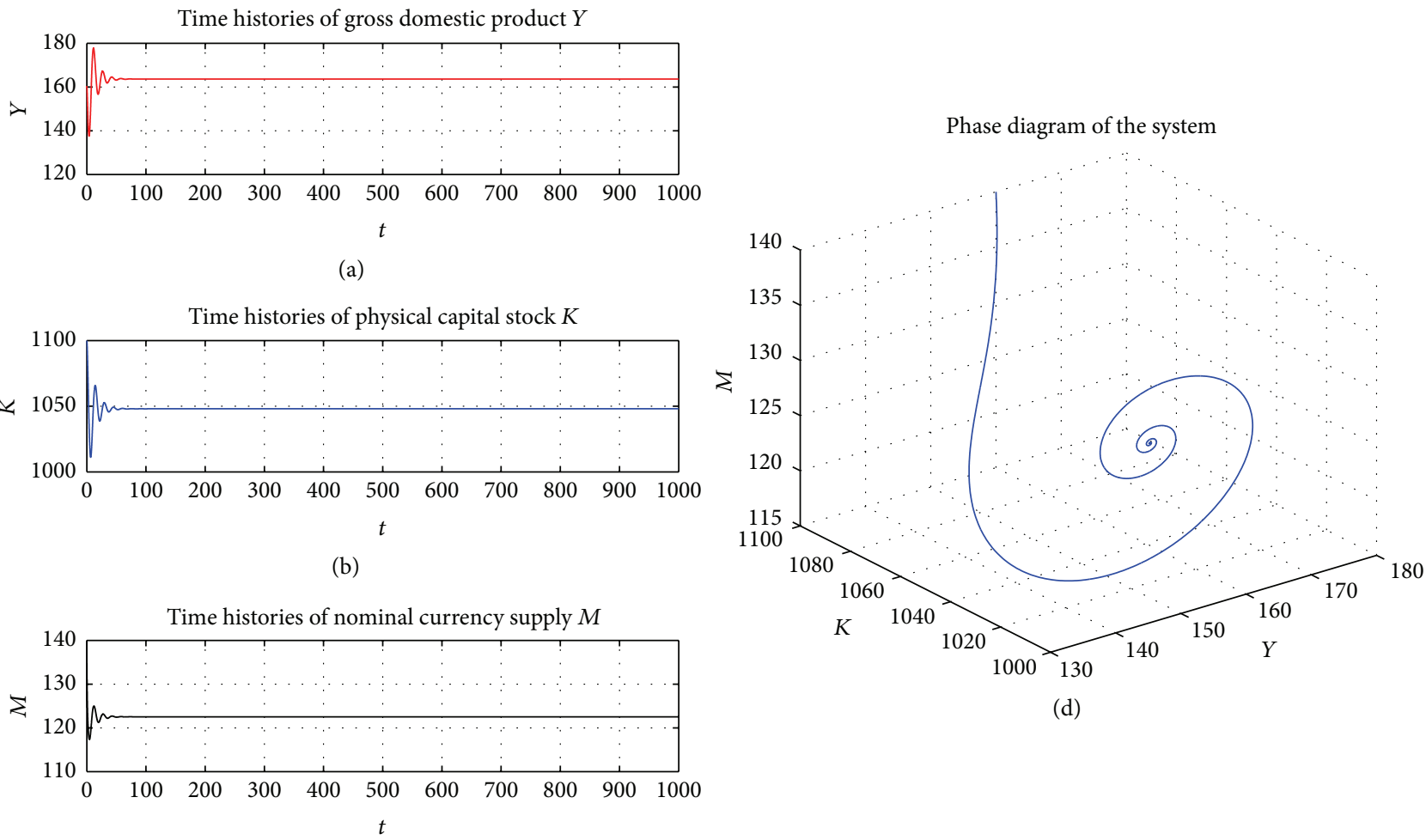

(d)

(c)

FIGURE 6: History and phase diagram of the system when $\alpha=1, \beta=1.5$.
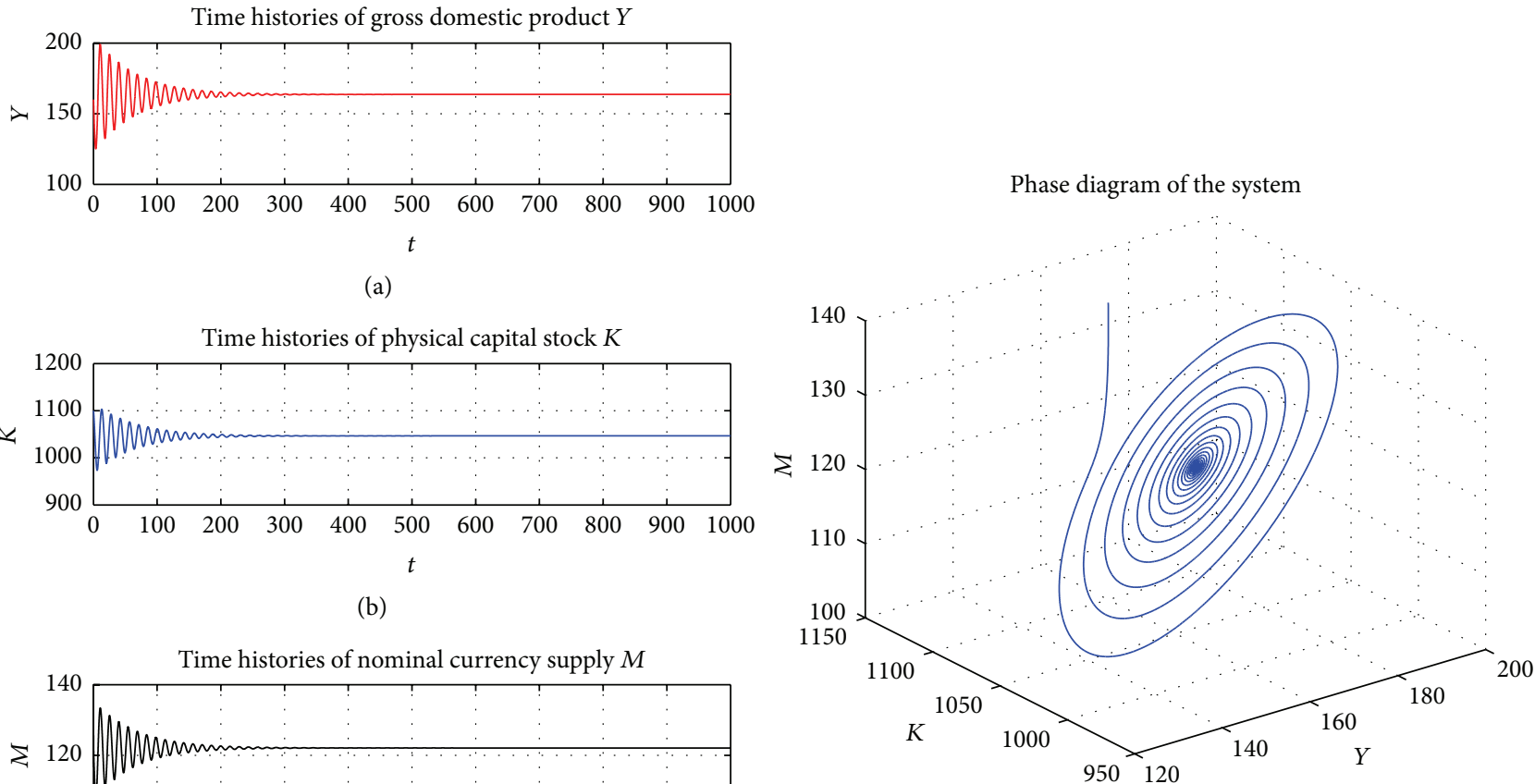

(a)

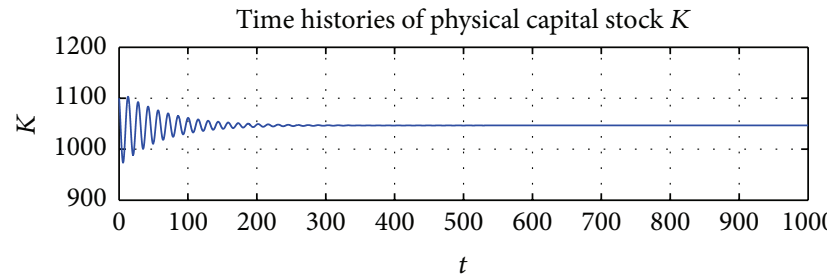

(b)

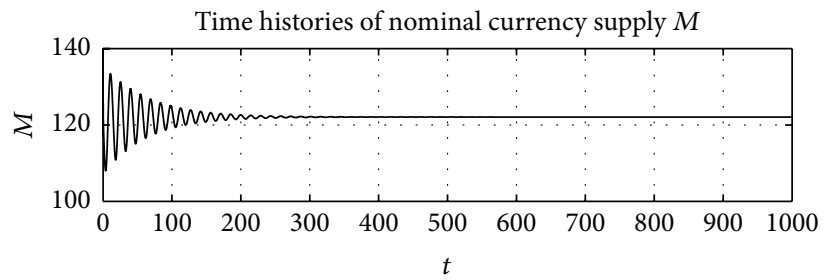

(d)

(c)

FIGURE 7: History and phase diagram of the system when $\alpha=1, \beta=6$. 

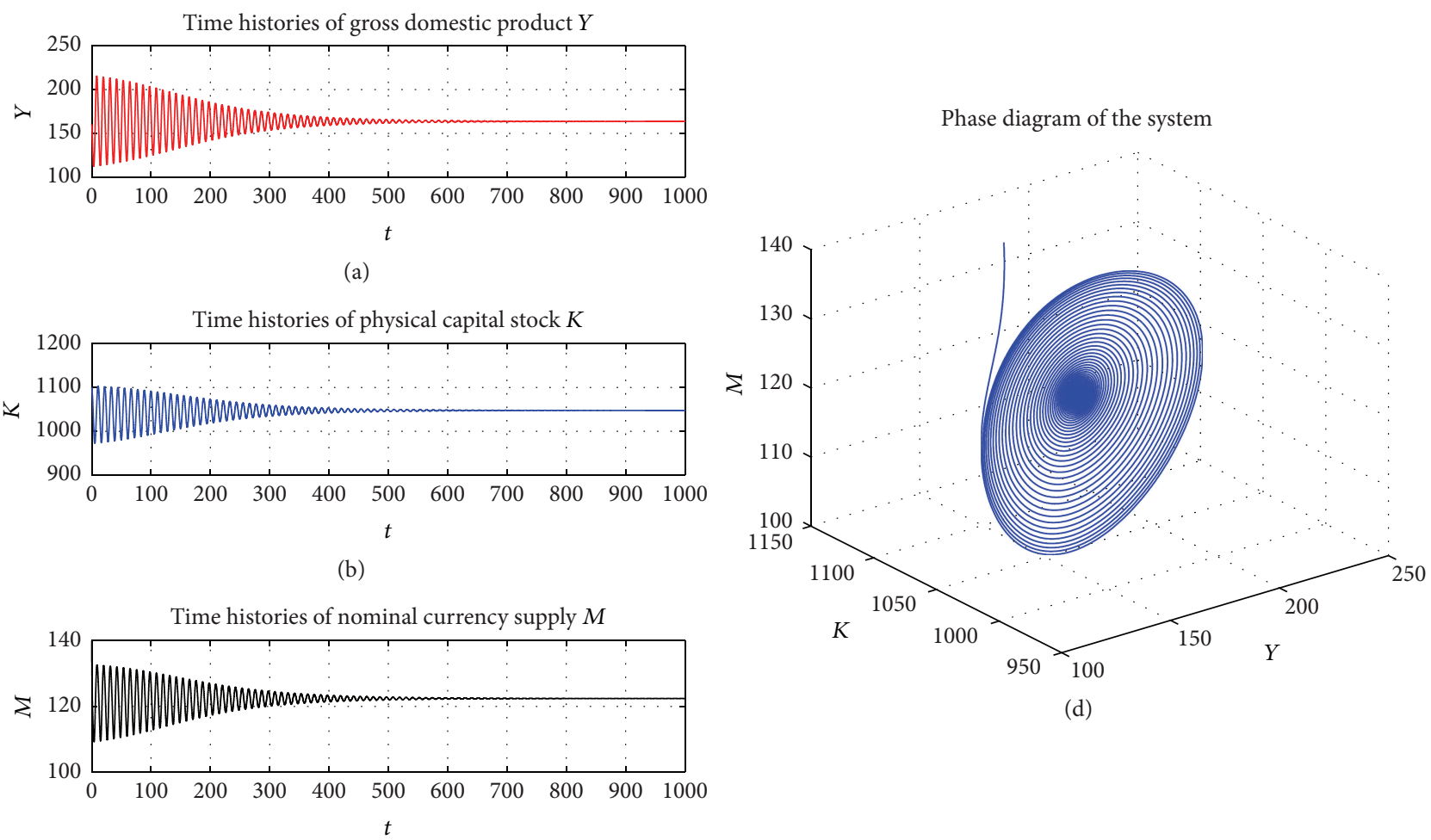

(d)

(c)

FIGURE 8: History and phase diagram of the system when $\alpha=2, \beta=1.9$.

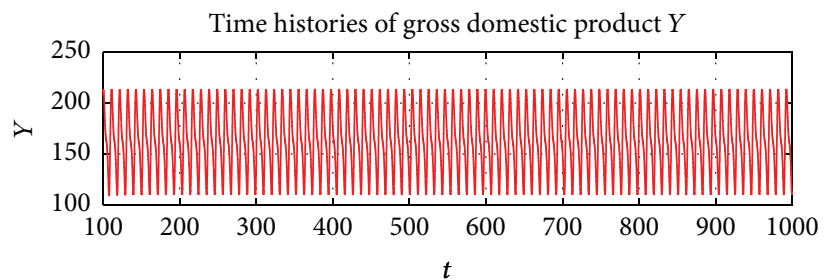

(a)

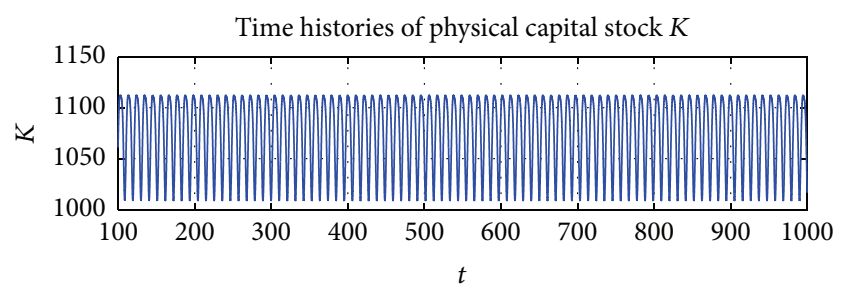

(b)
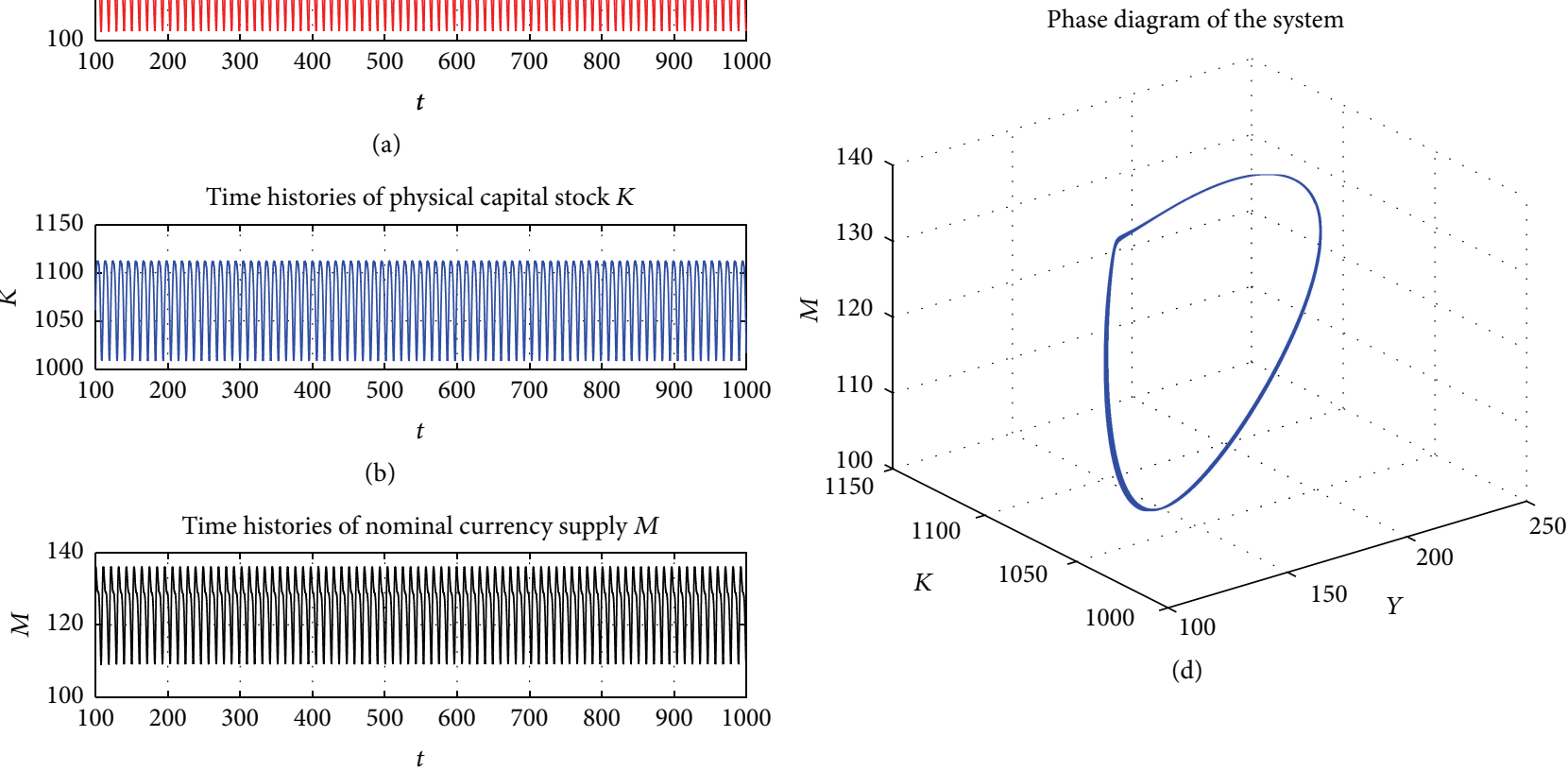

(d)

(c)

FIGURE 9: History and phase diagram of the system when $\alpha=2, \beta=2.5$. 


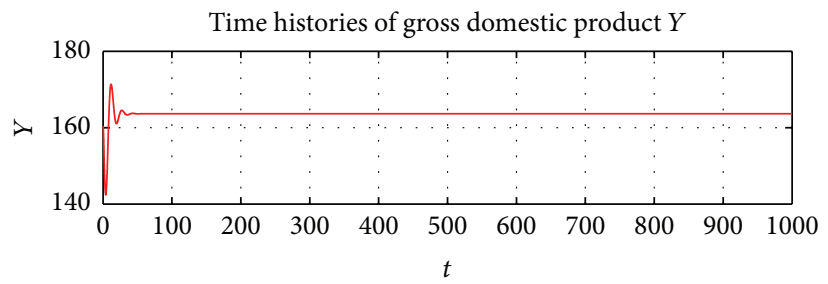

(a)

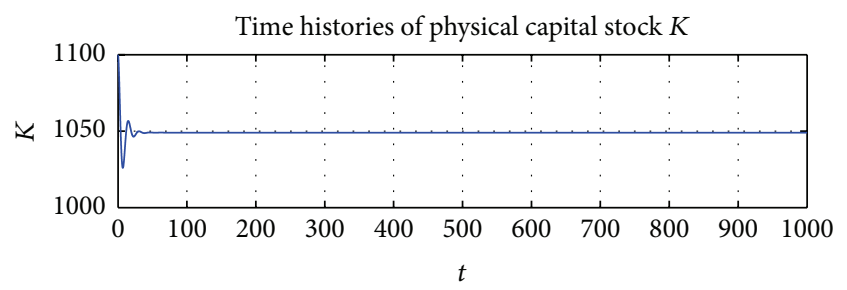

(b)
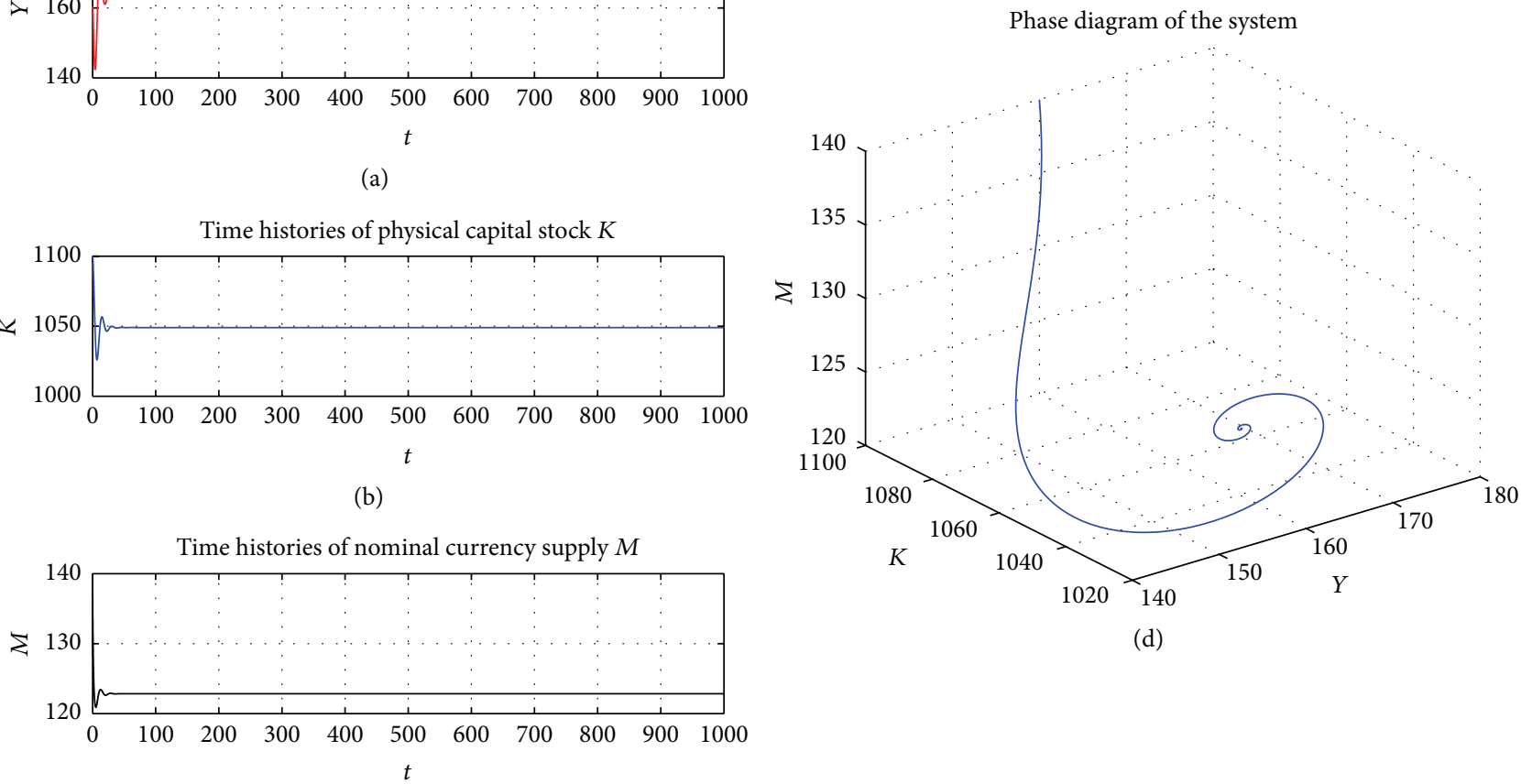

(d)

(c)

FIgURE 10: History and phase diagram of the system when $\alpha=1, \beta=1$.

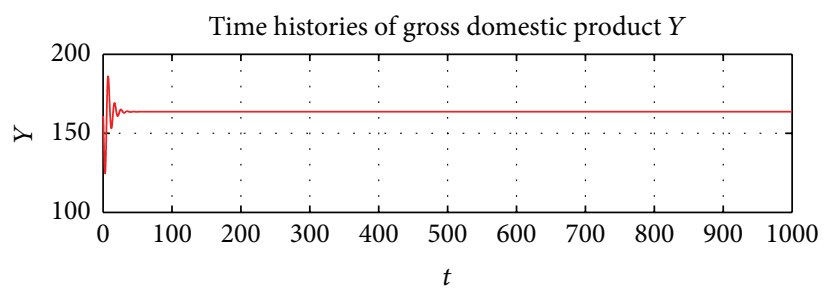

(a)

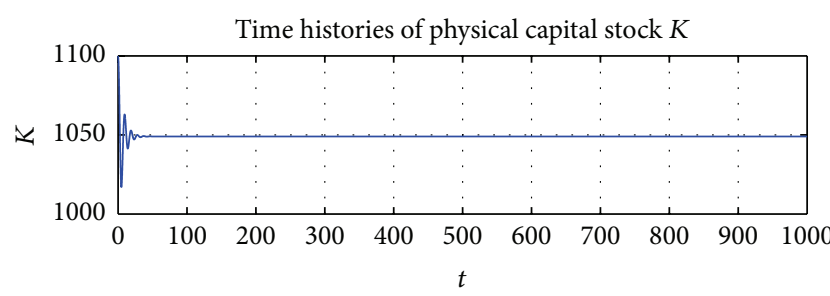

(b)
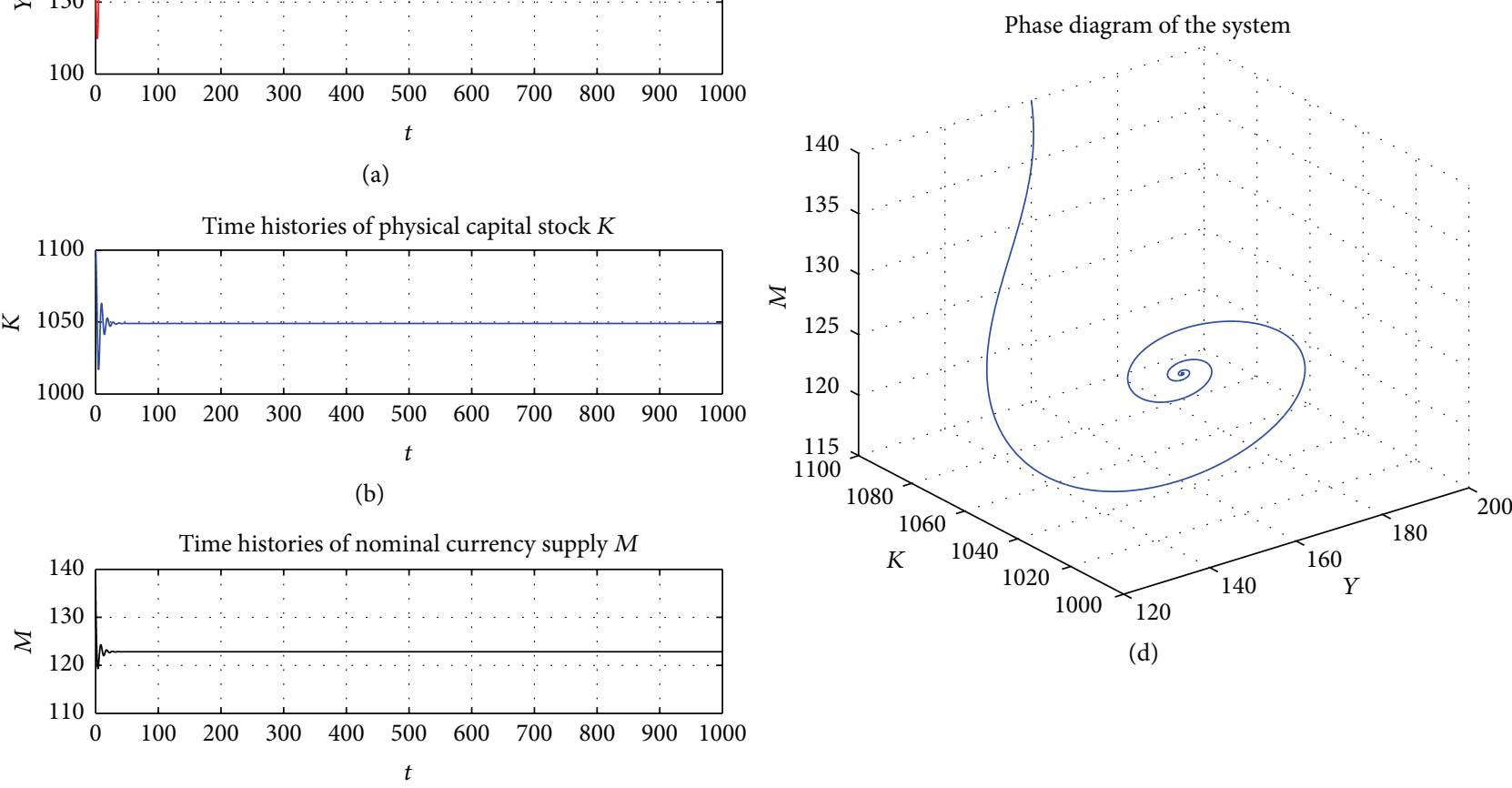

(d)

(c)

FIGURE 11: History and phase diagram of the system when $\alpha=3, \beta=1$. 

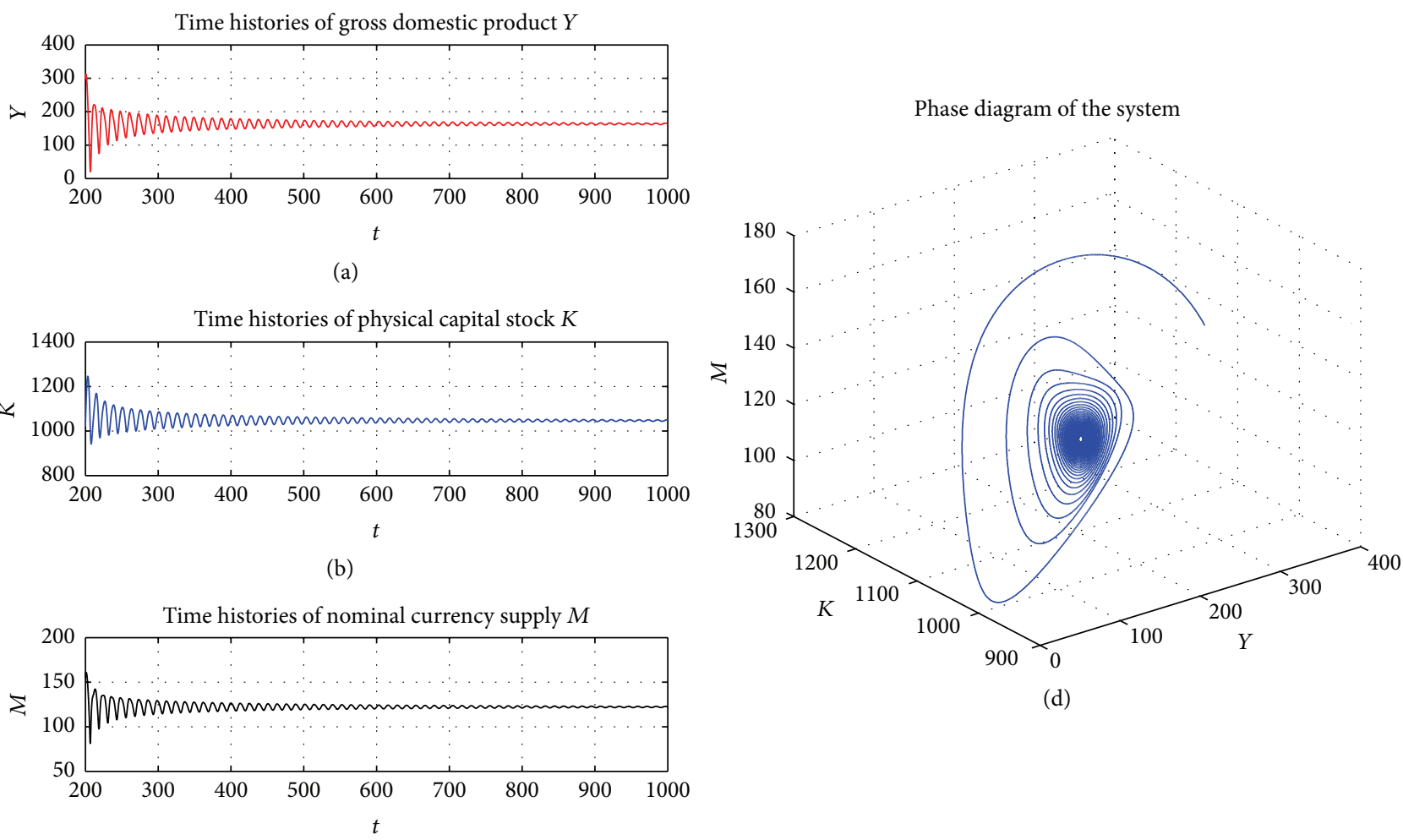

(d)

(c)

FIGURE 12: History and phase diagram of the system when $\alpha=1.4, \beta=3$.
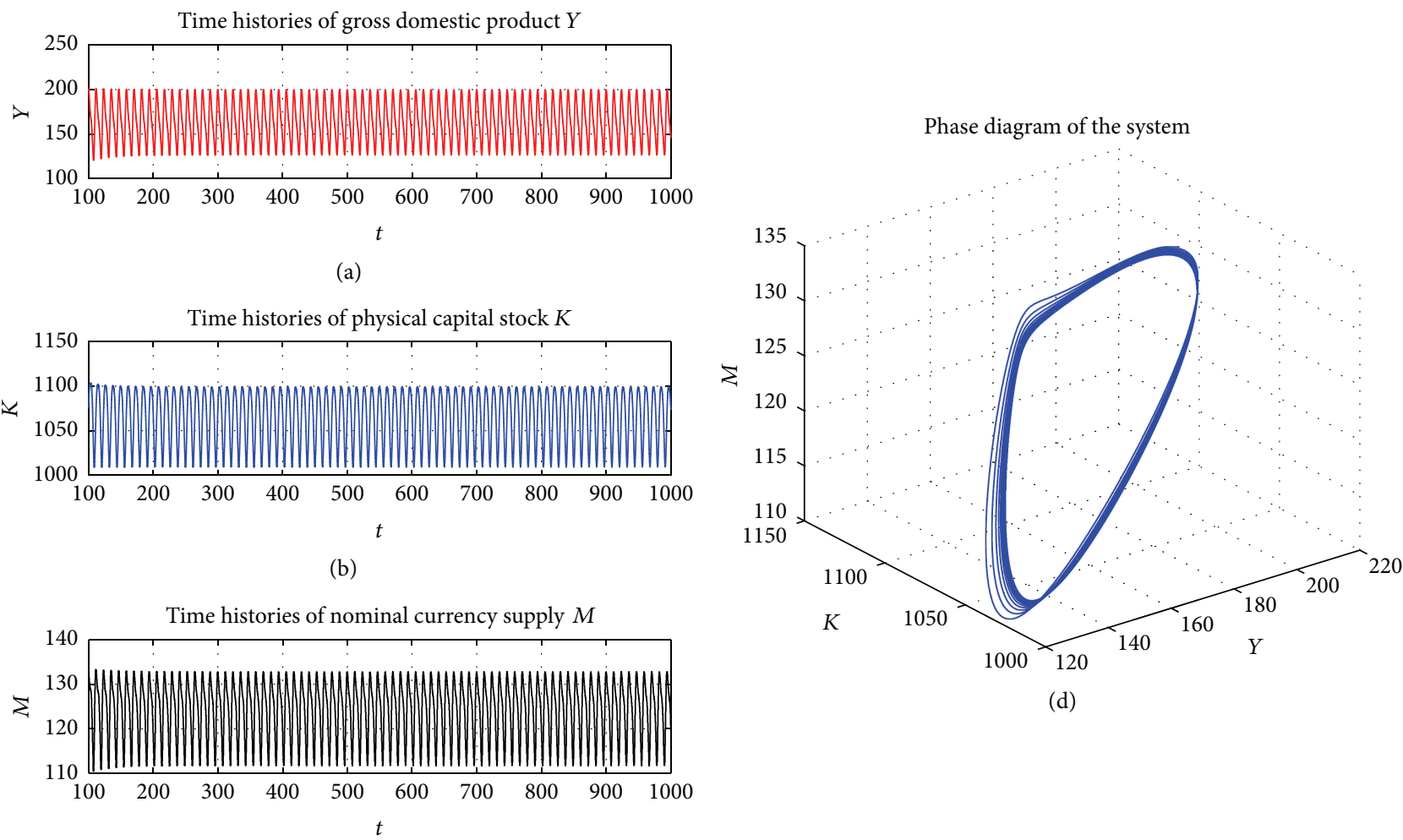

(d)

(c)

FIGURE 13: History and phase diagram of the system when $\alpha=1.6, \beta=3$. 


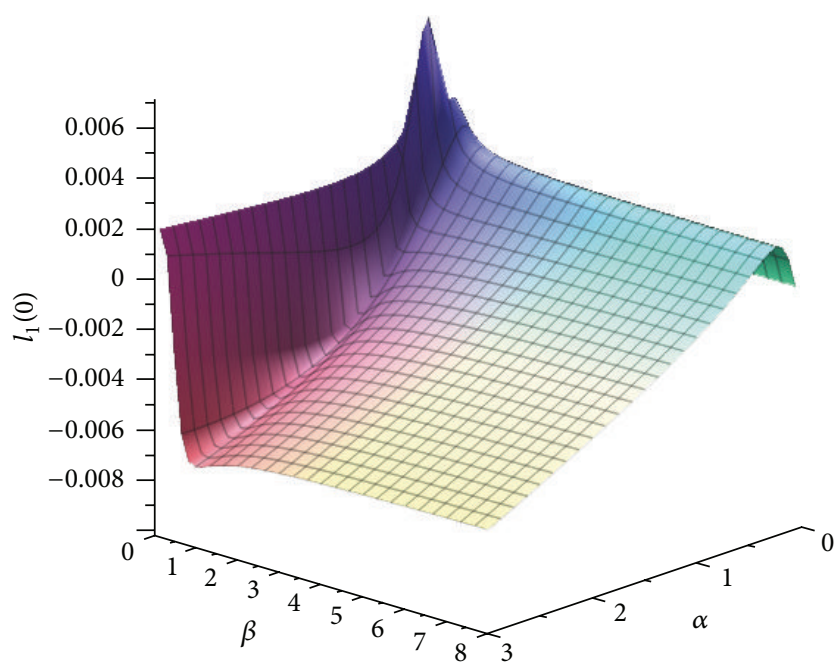

FIGURE 14: First Lyapunov coefficient diagram with parameters $\alpha, \beta$ changes.

bifurcation equation is $C-A B=0$ according to (27), and then

$$
\begin{gathered}
-0.32 \alpha \beta^{2}+0.23 \alpha \beta+0.3 \beta^{2}+0.15 \alpha+0.09 \beta \\
-0.023 \alpha^{2} \beta+0.037 \alpha^{2}=0 .
\end{gathered}
$$

The bifurcation curve corresponding to the certain equation is shown as in Figure 5.

We will investigate the existence of Hopf bifurcation of the system in the two aspects below.

(1) Fix $\alpha$ as a constant and observe the system status in company with the variations of the parameter $\beta$.

Let $\beta=10$; then $\alpha \approx \alpha_{0}=1.047$. It is easily proved that when $\alpha \in\left[1, \alpha_{0}\right)$, no matter how the parameter $\beta$ changes, the equilibrium point $E\left(Y^{*}, K^{*}, M^{*}\right)$ is always stable; when $\alpha \in\left[\alpha_{0}, 3\right], \beta_{0}(\alpha)$ exists that makes the system assume Hopf bifurcation, and the system passes into an unstable status. Let $\alpha=1<1.047$; observe the status of system when $\beta=1.5, \beta=$ 6 shown as in Figures 6 and 7.

Let $\alpha=1$, because $\alpha<\alpha_{0}=1.047$; as previously mentioned, when $\alpha \in\left[1, \alpha_{0}\right)$, despite the changes in parameter $\beta$, the system may vibrate for a short time and then converge to the equilibrium point $E\left(Y^{*}, K^{*}, M^{*}\right)$. Thus, as is shown in Figures 6 and $7, \beta=1.5$ and $\beta=6$ to observe the status of the system. It can be seen that the three variations in the system $Y, K, M$ rapidly converge to the equilibrium point, and the phase curve rotates around the equilibrium point at first and is rapidly attracted by the equilibrium. These phenomena are in agreement with the theory analysis.

When $\alpha=2>1.047$, according to (53), we get the critical value $\beta_{0}(2) \approx 2.01$ and then observe the status of the system with the parameters $\beta=1.9$ and $\beta=2.5$ shown in Figures 8 and 9.

Let $\alpha=2$, because $\alpha>\alpha_{0}=1.047$; then, according to the theory analysis, the critical value $\beta_{0}(2) \approx 2.01$. That is, when $\beta<2.01$, the system is stable near the equilibrium point shown in Figure 8. But when $\beta>2.01$, the system

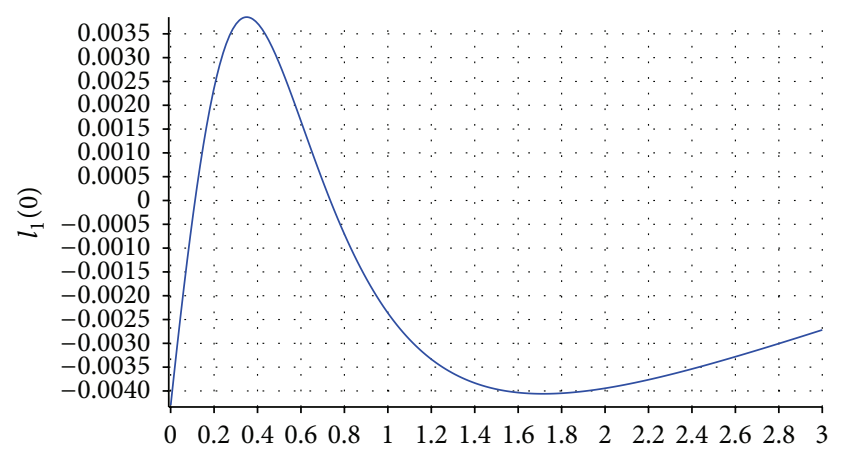

$\alpha$

FigURE 15: First Lyapunov coefficient diagram with parameters $\alpha, \beta$ changes.

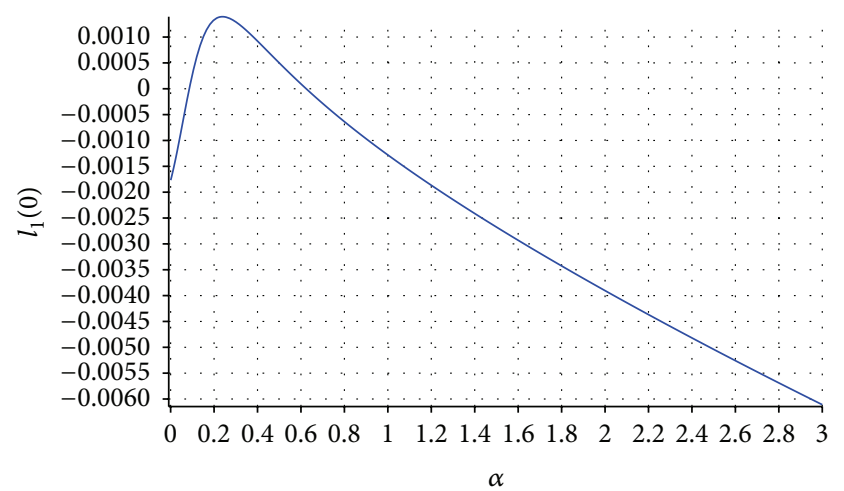

FIgURE 16: First Lyapunov coefficient diagram with parameters $\alpha, \beta$ changes.

becomes unstable and then a Hopf bifurcation and a limit cycle (business cycle) are generated as shown in Figure 9.

(2) Fix $\beta$ as a constant and observe the system status in company with the parameter $\alpha$.

Let $\alpha=1.5$; then $\beta \approx \beta_{0}=1.605$ and we can prove when $\beta \in\left[1.5, \beta_{0}\right)$, no matter the changes in parameter $\alpha$, the equilibrium $E\left(Y^{*}, K^{*}, M^{*}\right)$ will always be stable, but when $\beta \in\left[\beta_{0}, 10\right]$, a critical value $\alpha_{0}(\beta)$ exists, and the system generates a Hopf bifurcation. Then the system becomes unstable. If we take $\beta=1<1.605$ and observe the status of the system with parameters $\alpha=1$ and $\alpha=3$, the following is generated.

Let $\beta=1$, because $\beta<\beta_{0}=1.605$; as previously mentioned, when $\beta \in\left[1.5, \beta_{0}\right)$, despite the changes in parameter $\alpha$, the system may vibrate for a short time and then converge to the equilibrium point. Thus, as is shown in Figures 10 and 11 , we set $\alpha=1, \alpha=3$ to observe the status of the system. As a result it is found that the three variations of the system $Y, K, M$ rapidly converge to the equilibrium point, and the phase curve rotates at the equilibrium point at first and then moves rapidly to the equilibrium point. These phenomena are in agreement with the theoretical analysis. when $\beta=3>$ 1.605 , according to (53), the critical value $\beta_{0}(3) \approx 1.433$ was obtained. Then the status of the system with the parameters $\alpha=1.4$ and $\alpha=1.6$ as shown in Figures 12 and 13 becomes 


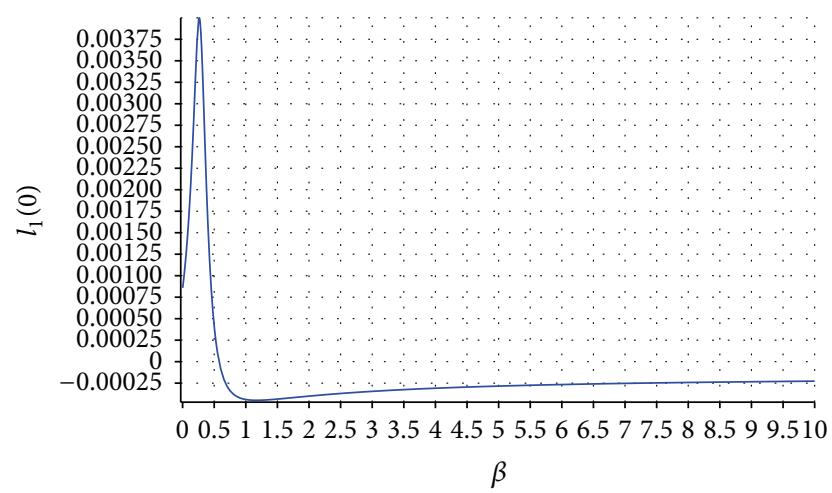

FIGURE 17: First Lyapunov coefficient diagram with parameters $\alpha, \beta$ changes.

unstable and then a Hopf bifurcation and a limited cycle (business cycle) are generated.

We continued to analyze the properties of the Hopf bifurcation generated by the system. In the analysis of the stability, the critical value was found, where the system varies from a stable status to an unstable status. In addition, the bifurcation equation showed that the system could generate Hopf bifurcation. To acquire the stability of the limited cycle generated by the Hopf bifurcation, the first Lyapunov coefficient must be calculated.

Figure 14 shows the first Lyapunov coefficient diagram with the changes in parameters $\alpha, \beta$. Based on the theory of nonlinear dynamics, when the first Lyapunov coefficient $l_{1}(0)<0$, the limited cycle generated by Hopf bifurcation is stable. Inversely, when $l_{1}(0)>0$, the limit cycle is unstable. As is shown in Figure 14, when $\alpha, \beta$ are near the origin point, the first Lyapunov coefficient is positive, but in other areas, the first Lyapunov coefficient is negative. Thus, we know that the limited cycle generated by the Hopf bifurcation is stable in most parts of the system. This result means that the system can generate a stable business cycle using Kaldorian model.

Parameter $\alpha$ was set as a constant to observe the variation in the first Lyapunov coefficient. As shown in Figure 15, it can be concluded when $\beta \in(0.085,0.601)$, the value of the first Lyapunov coefficient is positive, but when $\beta>1.5$, the first Lyapunov coefficient value is negative; that is to say, the economics meaning of this result is a stable business cycle and this phenomenon is the same when $\beta=5$ as shown in Figure 16.

Fixing the parameter as a constant to observe the variations of the first Lyapunov coefficient, as is shown in Figure 17, it can be concluded that when $\beta \in(0.135,0.734)$, the value of the first Lyapunov coefficient is positive, but when $\beta>0.734$, the first Lyapunov coefficient value is negative; that is to say, a stable business cycle is obtained, and this phenomenon is the same as the situation when $\alpha=2.1$ shown in Figure 18 .

Fixing the parameter $\beta$ as a constant to observe the variations of the first Lyapunov coefficient, as is shown in Figure 17, it can be concluded that when $\alpha \in(0.135,0.734)$, the value of the first Lyapunov coefficient is positive, but when

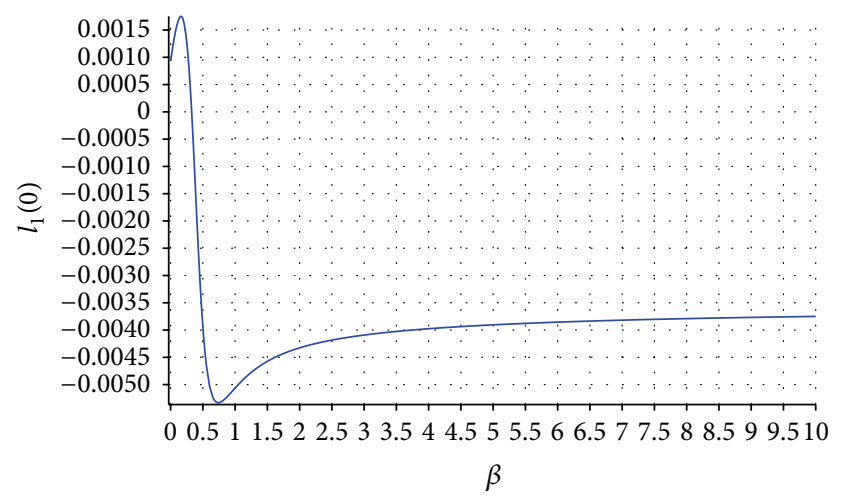

FIgURE 18: First Lyapunov coefficient diagram with parameters $\alpha, \beta$ changes.

$\alpha>1$, the first Lyapunov coefficient value is negative; that is to say, a stable business cycle is obtained, and this phenomenon is the same as the situation when $\alpha=2.1$ shown in Figure 18.

\section{Conclusion}

Nonlinear dynamic finance and economic models offer rich dynamic behavior. The results of the analysis of models are important from theoretical and practical perspectives whether the viewpoint is of a nonlinear system or implementation in macroeconomic policy.

In this reported study, a three-dimension nonlinear Kaldorian business cycle model was established based on a condition of fixed exchange rates. First, by solving a specific model, the resulting system had a unique equilibrium point. Second, using the speed of market regulation and the degree of capital movement as the variables in the system, the stability of the model is discussed and obtained when $\alpha$ and $\beta$ meet the relevant conditions described by (19); the equilibrium point is stable. Actually, the Kaldorian system in the condition of a fixed exchange rate is in equilibrium and the speed of market regulation $\alpha$ and the degree of capital movement $\beta$ have little influence on the status of the system. A decision maker can sustain the stability of an economic system by adjusting the speed of market regulation and the degree of capital movement. Third, by considering the existence of the Hopf bifurcation generated by the system, business cycle in the system was obtained where the parameters $\alpha$ and $\beta$ met Theorem 1 or Theorem 2. By analyzing the first Lyapunov coefficient, a stable limit cycle was obtained that was generated by the Hopf bifurcation, where the parameters of the system meet $l_{1}(0)<0$. Finally, a numerical simulation was provided to confirm the theoretical results. This study played an important role in the theoretical research of the model of the business cycle, and it is crucial for decision makers in the formulation of the macroeconomic policies.

\section{Competing Interests}

The authors declare that there are no competing interests regarding the publication of this paper. 


\section{References}

[1] M. Gallegati, L. Gardini, T. Puu, and I. Sushko, "Hicks' trade cycle revisited: cycles and bifurcations," Mathematics and Computers in Simulation, vol. 63, no. 6, pp. 505-527, 2003.

[2] T. Puu, "Chaos in business cycles," Chaos, Solitons \& Fractals, vol. 1, no. 5, pp. 457-473, 1991.

[3] T. Puu, Attractors, Bifurcations, and Chaos, Springer, Berline, Germany, 2000.

[4] T. Puu and I. Sushko, "A business cycle model with cubic nonlinearity," Chaos, Solitons \& Fractals, vol. 19, no. 3, pp. 597612, 2004.

[5] E. G. Mendoza, "Real business cycles in a small open economy," The American Economic Review, vol. 81, no. 4, pp. 797-818, 1991.

[6] A. C. Chu, G. Cozzi, C.-C. Lai, and C.-H. Liao, "Inflation, $\mathrm{R} \& \mathrm{D}$ and growth in an open economy," Journal of International Economics, vol. 96, no. 2, pp. 360-374, 2015.

[7] B. T. McCalluma and E. Nelson, "Nominal income targeting in an open-economy optimizing model," Journal of Monetary Economics, vol. 43, no. 3, pp. 553-578, 1999.

[8] B. De Paoli, "Monetary policy and welfare in a small open economy," Journal of International Economics, vol. 77, no. 1, pp. 11-22, 2009.

[9] T. Asada, V. Kalantonis, M. Markakis, and P. Markellos, "Analytical expressions of periodic disequilibrium fluctuations generated by Hopf bifurcations in economic dynamics," Applied Mathematics and Computation, vol. 218, no. 13, pp. 7066-7077, 2012.

[10] A. Matsumoto and F. Szidarovszky, "Continuous Hicksian trade cycle model with consumption and investment time delays," Journal of Economic Behavior and Organization, vol. 75, no. 1, pp. 95-114, 2010.

[11] M. Lavoie and W. Godley, "Kaleckian models of growth in a coherent stock-flow monetary framework: a kaldorian view," Journal of Post Keynesian Economics, vol. 24, no. 2, pp. 277-311, 2001.

[12] H.-W. Lorenz, Nonlinear Dynamical Economics and Chaotic Motion, vol. 334, Springer, New York, NY, USA, 1993.

[13] T. Asada, "Kaldorian dynamics in an open economy," Journal of Economics, vol. 62, no. 3, pp. 239-269, 1995. 


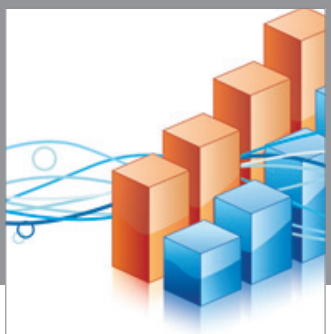

Advances in

Operations Research

vatem alat4

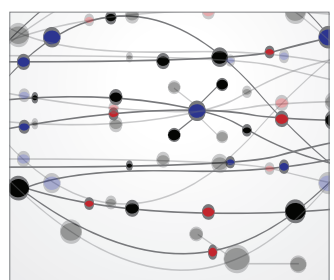

\section{The Scientific} World Journal
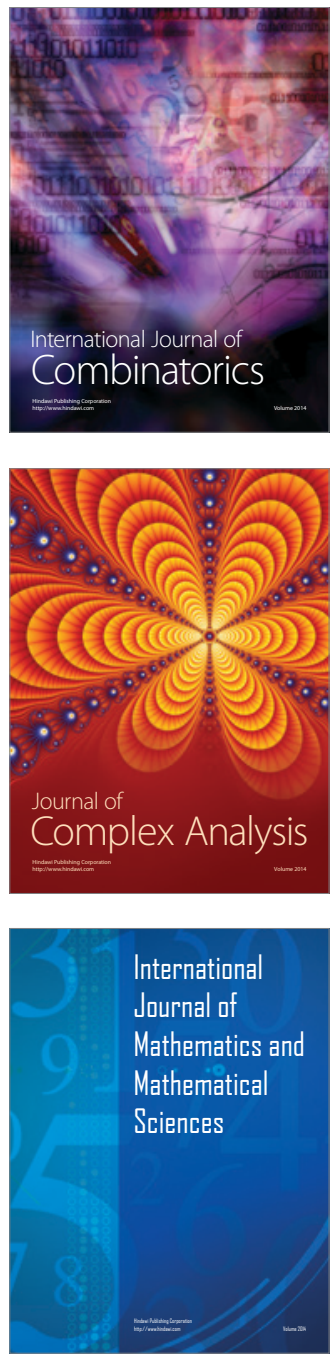
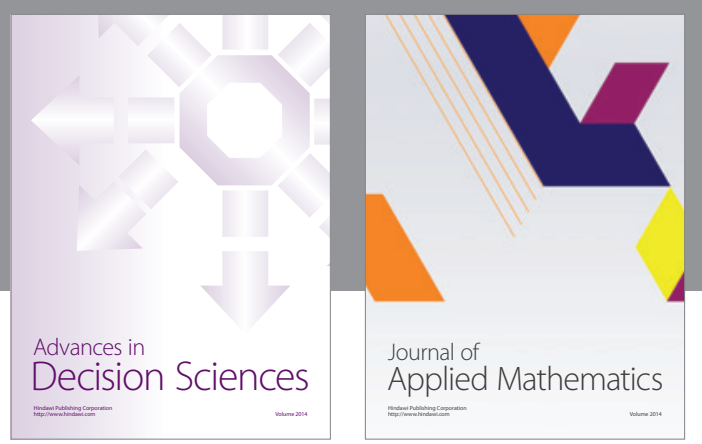

Algebra

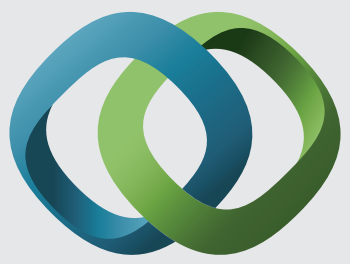

\section{Hindawi}

Submit your manuscripts at

http://www.hindawi.com
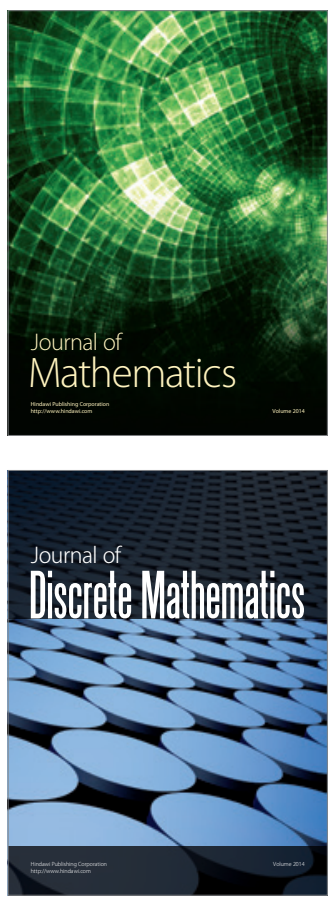

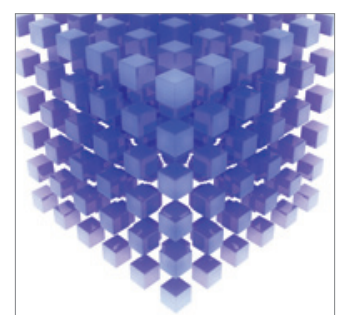

Mathematical Problems in Engineering
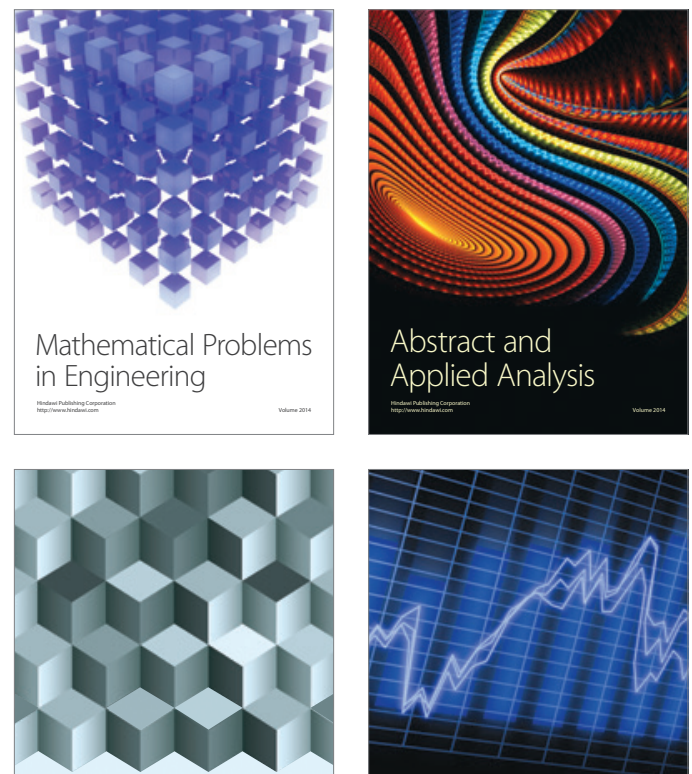

Journal of

Function Spaces

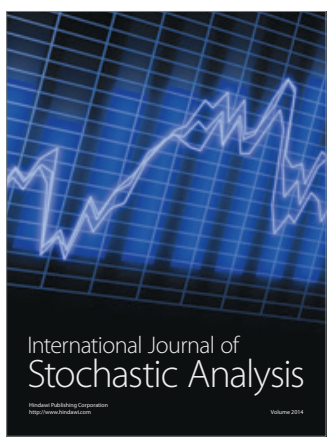

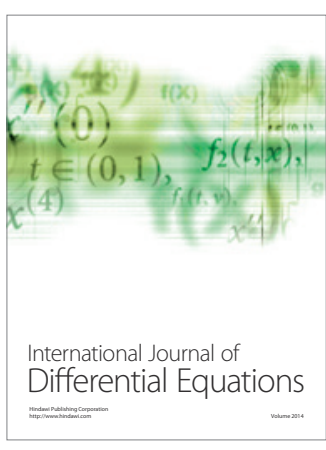
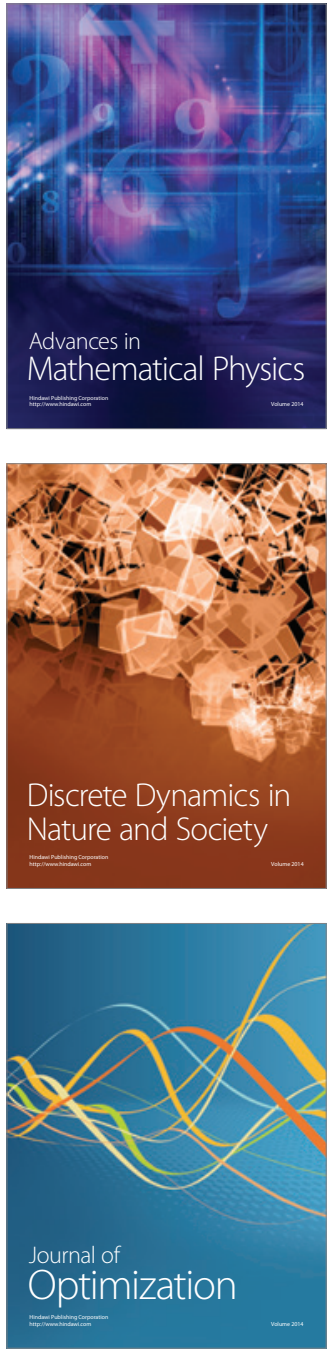